\title{
Subdivision of M1 Stage for De Novo Metastatic Breast Cancer to Better Predict Prognosis and Response to Primary Tumor Surgery
}

\author{
Caijin Lin, MMed*; Jiayi Wu, MD*; Shuning Ding, MMed*; Chihwan Goh, MMed; Lisa Andriani, MMed; \\ Shuangshuang Lu, MD; Kunwei Shen, MD, PhD; and Li Zhu, MD, PhD
}

\begin{abstract}
Background: Patients with de novo metastatic breast cancer (MBC) constitute a heterogeneous group with different clinicopathologic characteristics and survival outcomes. Despite controversy regarding its prognostic value, primary tumor surgery may improve survival for selected patients. Patients and Methods: Patients with de novo $M B C$ were identified using the SEER database and were then divided randomly into training and validation sets. A Fine-Gray competing risks model was developed to identify the variables associated with increased cancer-specific mortality in the training set. The M1 subdivision system was established based on the independent prognostic factors. Cumulative incidence curves were estimated and compared using Gray's test. Results: Involvement of brain or liver and number of metastatic sites were identified as independent prognostic factors in multivariate analysis. The $\mathrm{M} 1$ category was subdivided into 3 subcategories: $\mathrm{M} 1 \mathrm{a}$, single site involvement except brain and liver; M1b, liver involvement only, or multiple site involvement except brain and liver; and M1c, brain involvement regardless of number of metastatic sites, or liver and other sites involvement except brain (M1b vs M1a: subdistribution hazard ratio [SHR], $1.48 ; 95 \% \mathrm{Cl}$, 1.29-1.68; M1c vs M1a: SHR, 2.45; 95\% Cl, 2.18-2.75). Patients with the M1a subtype benefited most from primary tumor surgery in the adjusted competing risks model (M1a: SHR, 0.57; 95\% Cl, 0.48-0.67, M1b: SHR, 0.62; 95\% Cl, 0.47-0.83, and M1c: SHR, 0.59; 95\% Cl, $0.44-0.80$ ), whereas benefits conferred by treatment with chemotherapy alone increased with the upstaging of metastatic disease (M1a: SHR, 0.72; 95\% Cl, 0.62-0.83, M1b: SHR, 0.54; 95\% Cl, 0.44-0.68, and M1c: SHR, 0.53; 95\% Cl, 0.45-0.61). Conclusions: Subdivision of $\mathrm{M} 1$ stage facilitates prognosis prediction and treatment planning for patients with de novo MBC. Treatment offered should be decided in a coordinated multidisciplinary setting. Primary tumor surgery may play an important role in the management of selected patients.
\end{abstract}

J Natl Compr Canc Netw 2019;17(12):1521-1528 doi: $10.6004 /$ jnccn.2019.7332

Comprehensive Breast Health Center, Ruijin Hospital, Shanghai Jiao Tong University School of Medicine, Shanghai, People's Republic of China.

\section{Background}

Approximately $5 \%$ to $10 \%$ of patients with newly diagnosed breast cancer have distant metastases, with 5 -year cancer-specific mortality (CSM) ranging from $60 \%$ to $74 \%$ among different races/ethnicities. ${ }^{1}$ Median overall survival of patients with de novo metastatic breast cancer (MBC) is reported to be 12 to 32 months, suggesting that these patients constitute a heterogeneous group with a wide range of survival..$^{2-6}$ Consequently, it is not appropriate to assign these patients a "catch-all" classification.

Tumor $(\mathrm{T})$, node $(\mathrm{N})$, metastasis $(\mathrm{M})$ classification and staging of malignancy presents the anatomic extent of cancer. Of the 75 types of malignancy described by the TNM staging system, appropriately 20 include further subdivisions of the M1 category, such as prostate and lung cancers. ${ }^{7,8}$ However, the staging system for breast cancer uses only "M1" to describe metastatic disease. Therefore, all patients with de novo MBC are considered to have the same prognostic stage, regardless of histologic grade and HER2, estrogen receptor (ER), and progesterone receptor (PR) status. Hence, further subdivision of the M1 category for metastatic disease is of clinical importance for accurate prognosis prediction and personalized treatment planning. ${ }^{9-11}$

De novo MBC is considered incurable but treatable. Generally, systemic therapy is viewed as the mainstay of treatment. ${ }^{12}$ Primary tumor surgery is considered in selected patients, mostly in the palliative setting for those with impending complications, such as fungation and skin ulceration. ${ }^{13,14}$ Considerable controversy remains regarding the prognostic value of nonpalliative surgery for primary tumor. Two randomized trials have suggested caution in selecting patients for locoregional surgery due to little survival gain (ClinicalTrials.gov

See JNCCN.org for supplemental online content. 
identifiers: NCT00193778, NCT00941759), whereas a Turkish trial revealed potential benefits, especially in patients with solitary bone metastasis. ${ }^{5}$ However, the results are not conclusive enough due to inconsistency, and it remains uncertain which subgroup of patients would respond well to either monotherapies or combination therapy. To address this uncertainty, we performed a population-based study and subdivided patients with de novo MBC into different subcategories to stratify prognostication and help treatment planning.

\section{Patients and Methods}

\section{Database and Case Selection}

Population-based data were obtained from the SEER database, which covers approximately $28 \%$ of the US population. ${ }^{16}$ SEER collects and reports data on patient demographics, primary tumor sites and morphology, tumor stage at diagnosis, first treatment course, and survival outcomes.

Patients with de novo MBC diagnosed in 2010 through 2014 were identified using SEER*Stat software (version 8.3.5; seer.cancer.gov/seerstat). Breast cancer was identified using ICD-O-3 codes (C50.0-C50.6, C50.8, and C50.9). Only patients diagnosed with their first malignancy were included. All patients were $>18$ years of age. Patients with disease other than stage IV, unknown demographic characteristics and follow-up, or unknown involvement of bone, brain, liver, or lung, and those diagnosed at autopsy or via death certificate were excluded. Eligible patients were divided randomly into the training or validation sets using the basic $\mathrm{R}$ function sample().

\section{Statistical Analysis}

Pearson chi-square tests were used to analyze categorical variables. The main outcomes were CSM (breast cancer-specific death [BCSD]) and all-cause mortality. CSM was compared by estimating cumulative incidence curves according to Gray's test. ${ }^{17}$ A Fine-Gray regression model was developed to estimate subdistribution hazard ratios (SHRs) for CSM. ${ }^{18}$ Cumulative incidence curves of all-cause mortality were estimated based on the Kaplan-Meier method and compared using the log-rank test. The Cox proportional hazards model using a backward stepwise approach was built to estimate the hazard ratios (HR) for all-cause mortality. Patients in the training set were divided into several groups according to the combination of the independent prognostic factors for CSM. Cumulative incidence rates for CSM in each group were estimated and compared, using a 2-sided $P$ value of $<.001$ to ensure a significant difference in survival. Groups with significantly different prognosis were divided into different M1 subcategories.
In addition, multiple comparisons based on Gray's test and a Fine-Gray regression model were performed, with different treatment modalities used as the reference across M1 subcategories. To reduce the bias caused by no adjustment for use of radiotherapy (RT), endocrine therapy, and targeted anti-HER2 therapy, multiple comparisons were repeated in women who did not receive $\mathrm{RT}$ and in those with triple-negative breast cancer (TNBC) who did not receive RT.

All tests used 2 -sided $P$ values of $<.05$ suggesting statistical significance unless otherwise stated. Pearson Chi-square test and a Cox proportional hazards model were developed using SPSS Statistics, version 24 (IBM Corp). Kaplan-Meier method, Gray's test, and a FineGray regression model were performed using $\mathrm{R}$ version 3.4.3 (R Foundation for Statistical Computing).

\section{Results}

\section{Baseline Characteristics}

Of the 312,041 patients diagnosed with breast cancer, 8,582 were identified with de novo MBC, including 4,291 each in the training or validation sets. In both sets, the most frequently involved metastatic sites were bone, lung, liver, and brain, and clinicopathologic characteristics were well-balanced. Detailed baseline characteristics are outlined in Table 1 and supplemental eTables 1 , and 2 (available with this article at JNCCN.org).

\section{Association Between Characteristics of Distant Metastases and CSM}

Univariate analysis showed that involvement of brain, liver, or lung, and of multiple ( $>1$ or $>2$ ) metastatic sites was associated with increased CSM. Multivariable analysis showed that involvement of brain (SHR, 2.13; 95\% CI, 1.80-2.52; $P<.001$ ) and liver (SHR, 1.71; 95\% CI, 1.51-1.95; $P<.001$ ) were independent prognostic factors. After adjusting for the same parameters, involvement of multiple $(>1)$ metastatic sites remained a significant predictor of CSM (SHR, 1.28; 95\% CI, 1.11-1.47; $P<.001$ ) (supplemental eTable 3). Additionally, we found that involvement of multiple $(>1)$ metastatic sites was associated with increased CSM in the absence of brain involvement $(P<.001)$ (supplemental eFigure 1A-C). However, no significant difference was observed when brain was involved $(P>.05)$ (supplemental eFigure 1D-F).

\section{Subdivision of M1 Category}

Patients in the training set were divided into 5 groups according to the independent prognostic factors (see supplemental eTable 4 for comparisons among the 5 groups). Consequently, patients with de novo MBC were subdivided into 3 categories: Mla, involvement of a single site except brain or liver; M1b, liver involvement only, 


\section{Table 1. Baseline Patient Characteristics} $(\mathrm{N}=\mathbf{8}, \mathbf{5 8 2})$

\begin{tabular}{|c|c|c|c|}
\hline & $\begin{array}{c}\text { Training Set } \\
\mathbf{n}(\%)\end{array}$ & $\begin{array}{c}\text { Validation Set } \\
\text { n (\%) }\end{array}$ & $P$ Value \\
\hline Age, y & & & .370 \\
\hline$<41$ & $376(8.8)$ & $423(9.9)$ & \\
\hline $41-60$ & $1,850(43.1)$ & $1,836(42.8)$ & \\
\hline $61-80$ & $1,695(39.5)$ & $1,662(38.7)$ & \\
\hline$>80$ & $370(8.6)$ & $370(8.6)$ & \\
\hline Marital status & & & .965 \\
\hline Unmarried & $2,314(53.9)$ & $2,316(54.0)$ & \\
\hline Married & $1,977(46.1)$ & $1,975(46.0)$ & \\
\hline Histologic grade & & & .122 \\
\hline Well-differentiated & $306(7.1)$ & $322(7.5)$ & \\
\hline Moderately differentiated & $1,803(42.0)$ & $1,771(41.3)$ & \\
\hline Poorly differentiated & $2,135(49.8)$ & $2,170(50.6)$ & \\
\hline Undifferentiated & $47(1.1)$ & $28(0.7)$ & \\
\hline AJCC T category & & & .979 \\
\hline TO & $385(9.0)$ & $377(8.8)$ & \\
\hline $\mathrm{T} 1$ & $457(10.7)$ & $455(10.6)$ & \\
\hline $\mathrm{T} 2$ & $1,309(30.5)$ & $1,326(30.9)$ & \\
\hline T3 & $720(16.8)$ & $703(16.4)$ & \\
\hline T4 & $1,420(33.1)$ & $1,430(33.3)$ & \\
\hline AJCC $N$ category & & & .657 \\
\hline No & $1,158(27.0)$ & $1,155(26.9)$ & \\
\hline N1 & $1,927(44.9)$ & $1,892(44.1)$ & \\
\hline N2 & $515(12.0)$ & $552(12.9)$ & \\
\hline N3 & $691(16.1)$ & $692(16.1)$ & \\
\hline Subtype & & & .099 \\
\hline HoR +/HER2- & $2,554(59.5)$ & $2,491(58.1)$ & \\
\hline HoR +/HER2 + & $775(18.1)$ & $744(17.3)$ & \\
\hline HoR-/HER2+ & $387(9.0)$ & 409 (9.5) & \\
\hline Triple-negative & $575(13.4)$ & $647(15.1)$ & \\
\hline Bone involvement & & & .338 \\
\hline No & $1,343(31.3)$ & $1,302(30.3)$ & \\
\hline Yes & $2,948(68.7)$ & $2,989(69.7)$ & \\
\hline Brain involvement & & & .560 \\
\hline No & $3,987(92.9)$ & $3,973(92.6)$ & \\
\hline Yes & $304(7.1)$ & $318(7.4)$ & \\
\hline Liver involvement & & & .885 \\
\hline No & $3,108(72.4)$ & $3,102(72.3)$ & \\
\hline Yes & $1,183(27.6)$ & $1,189(27.7)$ & \\
\hline Lung involvement & & & .231 \\
\hline No & $2,872(66.9)$ & $2,924(68.1)$ & \\
\hline Yes & $1,419(33.1)$ & $1,367(31.9)$ & \\
\hline
\end{tabular}

Abbreviation: HoR, hormone receptor.

\begin{tabular}{|c|c|c|c|}
\hline & $\begin{array}{c}\text { Training Set } \\
\mathbf{n}(\%)\end{array}$ & $\begin{array}{c}\text { Validation Set } \\
\text { n (\%) }\end{array}$ & $P$ Value \\
\hline \multicolumn{4}{|l|}{ Number of metastatic sites } \\
\hline Binary classifications 1 & & & .837 \\
\hline 1 site & $2,854(66.5)$ & $2,845(66.3)$ & \\
\hline$>1$ site & $1,437(33.5)$ & $1,446(33.7)$ & \\
\hline Binary classifications 2 & & & .648 \\
\hline 1 or 2 sites & $3,919(91.3)$ & 3,907 (91.1) & \\
\hline$>2$ sites & $372(8.7)$ & $384(8.9)$ & \\
\hline Treatment modality & & & .843 \\
\hline None & $1,326(30.9)$ & $1,345(31.3)$ & \\
\hline Surgery only & $485(11.3)$ & 469 (10.9) & \\
\hline Chemotherapy only & $1,376(32.1)$ & $1,397(32.6)$ & \\
\hline Surgery + chemotherapy & $1,104(25.7)$ & $1,080(25.2)$ & \\
\hline
\end{tabular}

Abbreviation: HoR, hormone receptor.

or involvement of multiple sites except brain or liver; M1c, brain involvement regardless of number of metastatic sites, or involvement of liver and other sites except brain. Both the 3-year CSM and all-cause mortality rates increased across M1 subcategories (Figure 1A, 1D). In the adjusted competing risk model, the association of M1 subcategories with CSM remained significant (M1b vs M1a: SHR, 1.48; 95\% CI, 1.29-1.68; $P<.001$, and M1c vs M1a: SHR, 2.45; 95\% CI, 2.18-2.75; $P<.001$ ) (Table 2 and supplemental eTable 5). Additionally, the M1 subcategories were also associated with increased all-cause mortality (M1b vs M1a: SHR, 1.47; 95\% CI, 1.30-1.66; $P<.001$, and Mlc vs Mla: SHR, 2.38, 95\% CI, 2.14-2.65; $P<.001$ ) (Table 2 and supplemental eTable 6).

\section{Validation of M1 Category Subdivision}

Validation of the M1 category subdivision system is described in supplemental eAppendix 1, eFigure 2, and eTables 5-8.

Associations of Treatment Modality and M1 Subcategories With Survival Outcomes

Cumulative incidence curves show the mortality reduction by primary tumor surgery, chemotherapy, or combination therapy across M1 subcategories (Figure 2A-C).

When the monotherapies were compared with no treatment in the whole population, primary tumor surgery remained a favorable prognostic factor in the adjusted competing risk model, especially for patients with Mla disease (Mla: SHR, 0.57; 95\% CI, 0.48-0.67; $P<.001$, M1b: SHR, 0.62; 95\% CI, 0.47-0.83; $P=.001$, and M1c: SHR, 0.59; 95\% CI, 0.44-0.80; $P<.001$ ) (Table 3). Furthermore, patients with Mla disease benefited less 
A

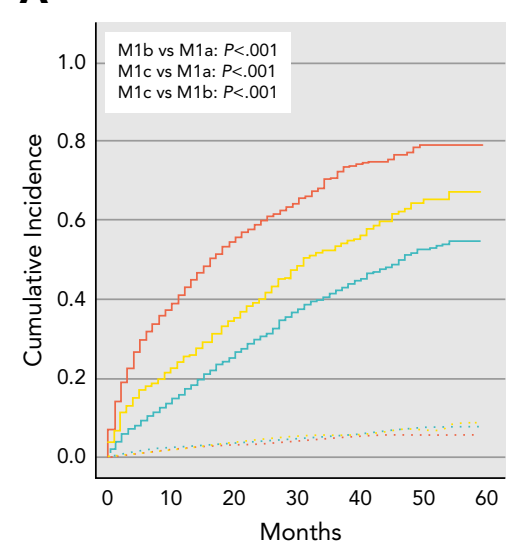

D

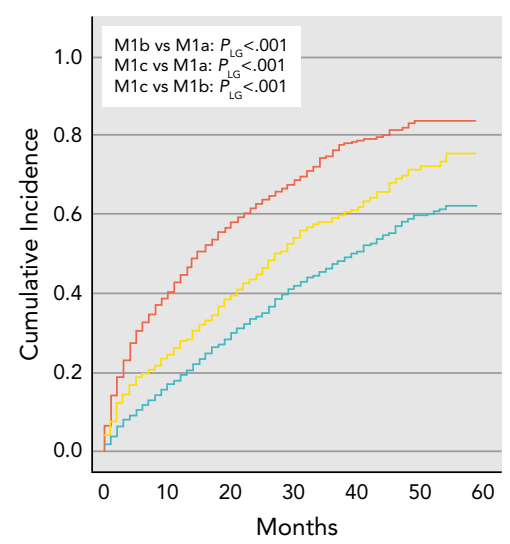

B

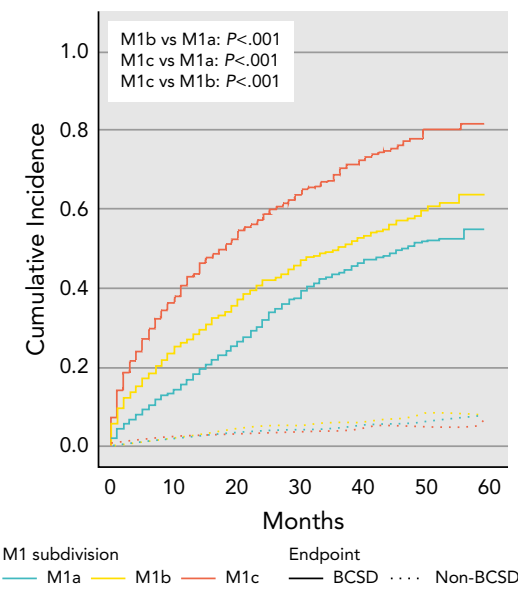

E

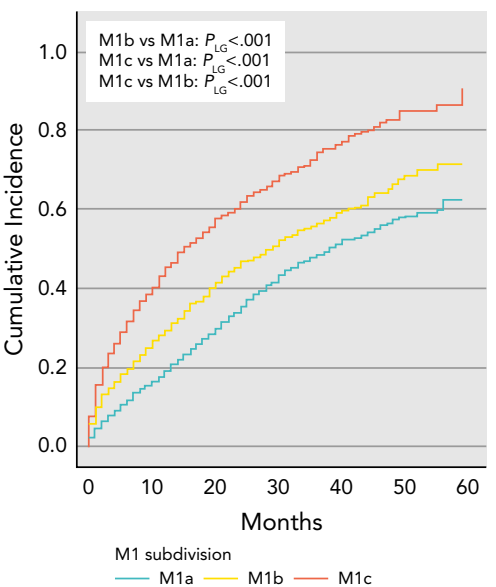

C

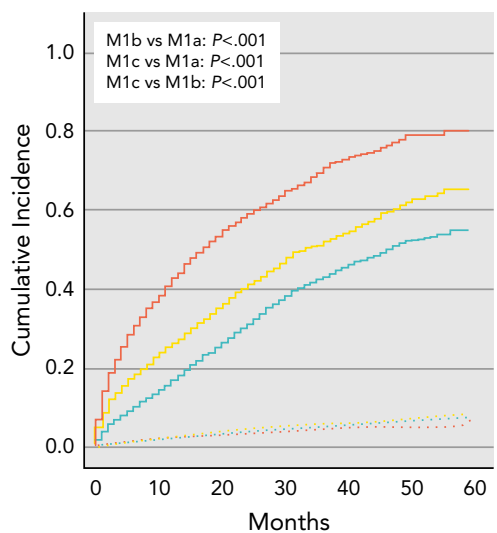

$\mathbf{F}$

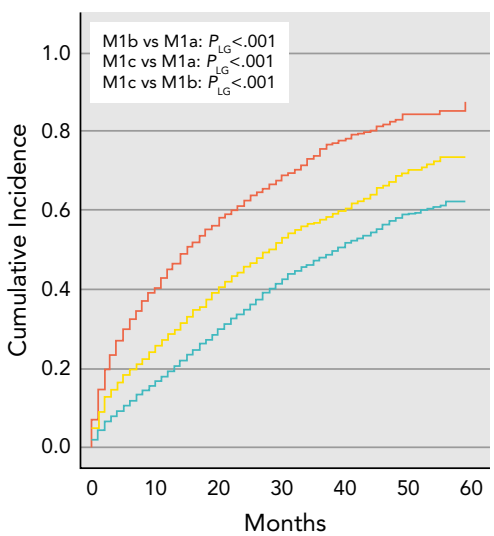

Figure 1. Cumulative incidence curves of $(A-C)$ cancer-specific and $(D-F)$ all-cause mortality for different $M 1$ subcategories in the $(A, D)$ training, $(B, E)$ validation, and $(C, F)$ whole sets. $P$ values were estimated and compared based on Gray's test (A-C) or log-rank test (D-F). Abbreviations: BCSD, breast cancer-specific death; LG, log-rank.

from chemotherapy alone than those with M1b or M1c disease (Mla: SHR, 0.72; 95\% CI, 0.62-0.83; $P<.001$, M1b: SHR, 0.54; 95\% CI, 0.44-0.68; $P<.001$, and M1c: SHR, 0.53; 95\% CI, $0.45-0.61 ; P<.001)$. Specifically, no significant difference in survival was seen between patients treated with surgery only and those treated with chemotherapy only, with the exception of patients with Mla disease, among whom those receiving chemotherapy only presented a worse prognosis (SHR, 1.27; 95\% CI, 1.06-1.51; $P=.008$ ). As for the combined therapy, those receiving the combination exhibited the lowest SHR across M1 subcategories (M1a: SHR, 0.39; 95\% CI, 0.34-0.46; $P<.001$, M1b: SHR, 0.46; 95\% CI, 0.36-0.58, $P<.001$, and M1c: SHR, 0.32; 95\% CI, 0.26-0.38; $P<.001)$. However, for patients classified as $\mathrm{Mlb}$, the combination therapy provided similar benefits to monotherapies (combination vs surgery only: SHR, 0.73 ; $95 \%$ CI, $0.54-1.00 ; P=.048$; combination vs chemotherapy only: SHR, 0.84; 95\% CI, 0.68-1.03; $P=.094$ ).
Similar results were observed in patients with or without TNBC who did not receive RT. Cumulative incidence curves are presented in supplemental eFigure 3. Among patients who did not receive RT $(n=5,584)$, those classified as M1c who received chemotherapy only had a better prognosis than those undergoing surgery only (SHR, $0.69 ; 95 \% \mathrm{CI}, 0.49-0.97 ; P=.035$ ) (supplemental eTable 9). In patients with TNBC not receiving RT $(n=808)$, those receiving locoregional surgery presented similar prognosis to treatmentnaïve patients (M1a: SHR, 0.94; 95\% CI, 0.56-1.59; $P=.824$, M1b: SHR, 0.78; 95\% CI, 0.29-2.12; $P=.629$, and Mlc: SHR, $1.50 ; 95 \% \mathrm{CI}, 0.75-3.02 ; P=.251$ ). Compared with surgery, chemotherapy conferred a greater survival benefit across $\mathrm{M} 1$ subcategories (M1a: SHR, 0.65, 95\% CI, 0.44-0.96; $P=.031$, Mlb: SHR, 0.34; 95\% CI, 0.16-0.74; $P=.006$, and Mlc: SHR, 0.32; 95\% CI, $0.16-0.64 ; P=.001)$. 
Table 2. Multivariate Analysis of Cancer-Specific and All-Cause Mortality ${ }^{\mathrm{a}}$

\begin{tabular}{|c|c|c|c|c|c|c|}
\hline & \multicolumn{2}{|c|}{ Training Set } & \multicolumn{2}{|c|}{ Validation Set } & \multicolumn{2}{|c|}{ Whole Set } \\
\hline \multicolumn{7}{|c|}{ Part I: cancer-specific mortality } \\
\hline M1a & Ref & & Ref & & Ref & \\
\hline M1b & $1.48(1.29-1.68)$ & $<.001$ & $1.32(1.17-1.51)$ & $<.001$ & $1.39(1.27-1.53)$ & $<.001$ \\
\hline M1c & $2.45(2.18-2.75)$ & $<.001$ & $2.15(1.92-2.42)$ & $<.001$ & $2.29(2.11-2.48)$ & $<.001$ \\
\hline M1a & Ref & & Ref & & Ref & \\
\hline M1b & $1.47(1.30-1.66)$ & $<.001$ & $1.35(1.20-1.53)$ & $<.001$ & $1.41(1.29-1.53)$ & $<.001$ \\
\hline M1c & $2.38(2.14-2.65)$ & $<.001$ & $2.09(1.88-2.33)$ & $<.001$ & $2.22(2.06-2.41)$ & $<.001$ \\
\hline
\end{tabular}

Abbreviation: SHR, subdistribution hazard ratio.

${ }^{a}$ Adjusted variables included age, race/ethnicity, marital status, histologic grade, AJCC T \& N category, molecular subtype, and treatment modality. For detailed data, see supplemental eTables 5 and 6 .

In addition, comparisons between lumpectomy and mastectomy are presented in supplemental eTable 10. No significant difference in mortality was observed.

\section{Discussion}

This study focused on the association between anatomic extent of metastatic disease and CSM in patients with de novo MBC. We found that involvement of liver and brain and of multiple metastatic sites were independently associated with CSM. We also further subdivided patients with significantly different prognoses into Mla, M1b, or M1c subcategories, and found that cumulative incidence rates of both CSM and all-cause mortality were significantly different across M1 subcategories. Our findings indicate that selected patients might benefit from aggressive locoregional treatment and that subdivision of M1 stage may guide individualized treatment planning.
Consistent with previous research, ${ }^{19}$ we found that brain and liver involvement were each independently associated with an unfavorable prognosis for patients with de novo MBC. In our study, the number of sites involved proved to be an independent predictor for CSM, which was accordant with the findings of many previous studies, which reported HRs ranging from 1.45 (95\% CI, 1.35-1.55) to $1.56(95 \%$ CI, $1.02-2.40){ }^{4,5,20,21}$ The results of both the previous study and the present study indicated that metastatic sites and the number of sites involved might be key parameters for risk stratification. In autopsy studies, patients with brain metastases were reported as more likely to have multiple sites involved. ${ }^{22}$ Interestingly, no significant difference in survival was observed between patients with single and multiple metastatic sites in the presence of brain involvement in our study. Hence, we established an M1 subdivision system and divided patients with boneonly or lung-only metastases into the Mla category due
A

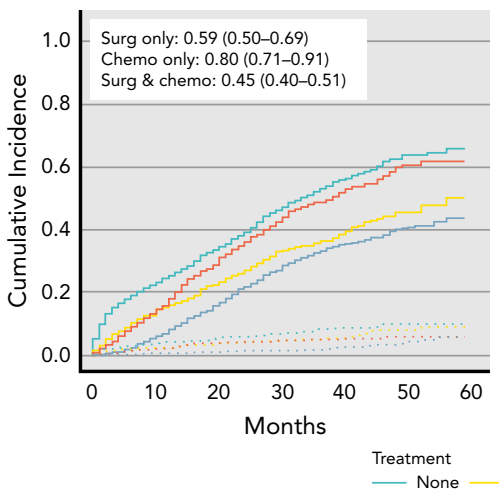

B

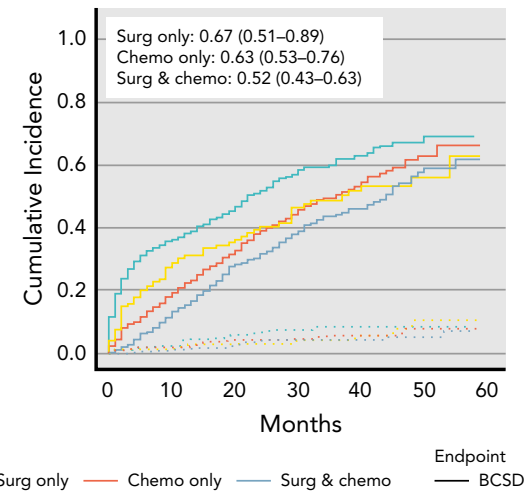

C

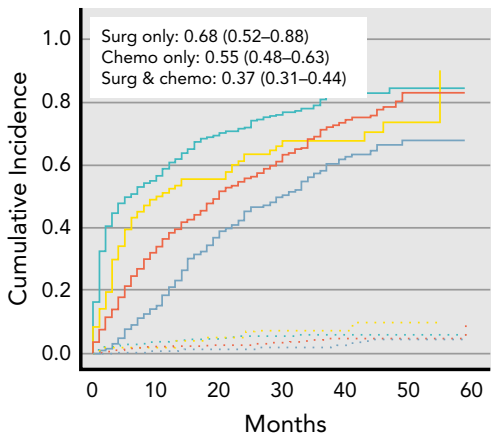

Non-BCSD

Figure 2. Cumulative incidence curves of cancer-specific mortality stratified by treatment modality in patients with (A) M1a, (B) M1b, and (C) M1c disease in the whole set. Patients receiving no treatment were used as the reference category and data are presented as SHR ( $95 \% \mathrm{Cl})$. Abbreviations: BCSD, breast cancer-specific death; chemo, chemotherapy; SHR, subdistribution hazard ratio; surg, surgery. 
Table 3. Association of Cancer-Specific Mortality With Treatment Modality ${ }^{a}$

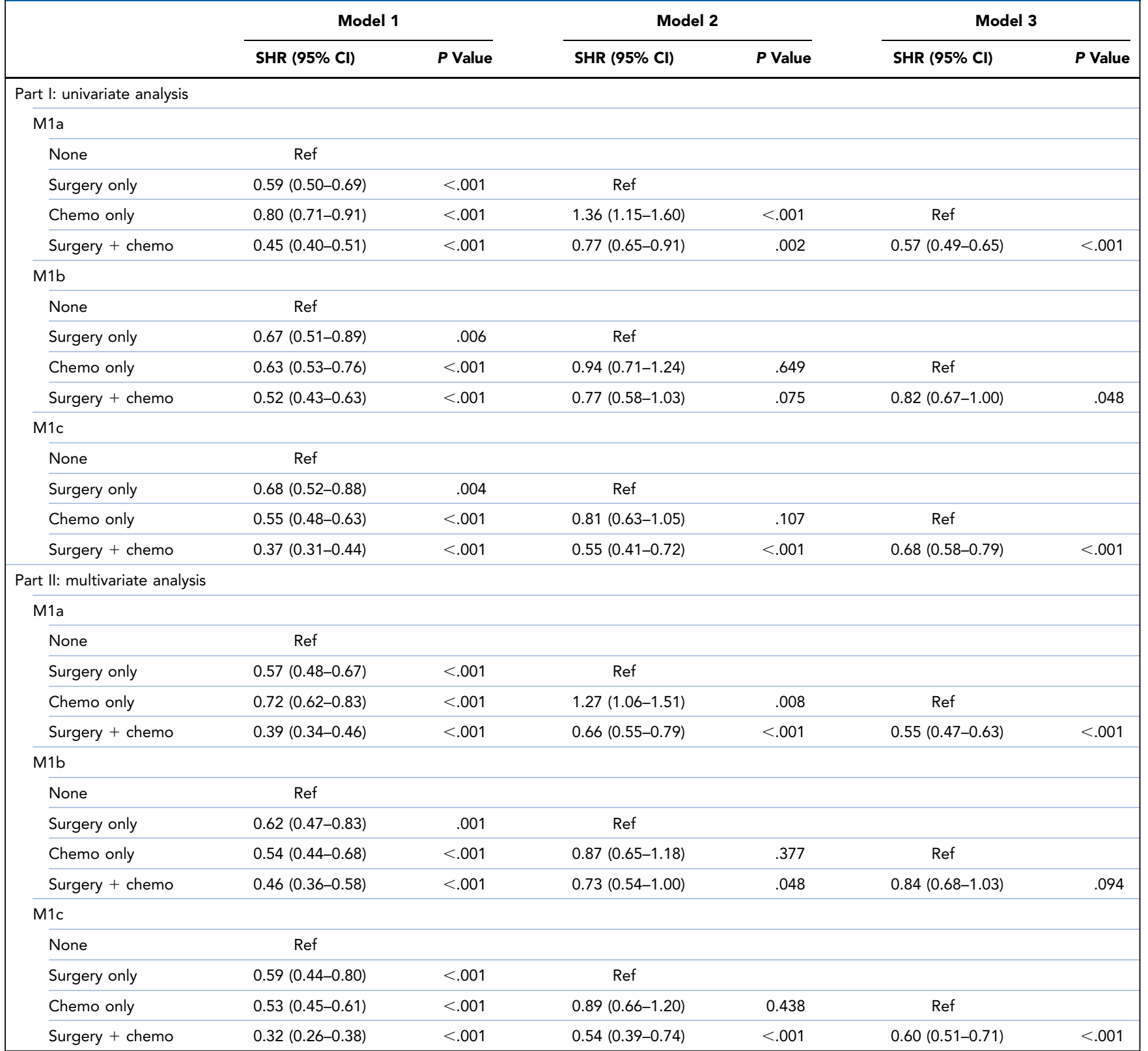

Abbreviations: Chemo, chemotherapy; SHR, subdistribution hazard ratio.

aln both the univariate and multivariate analyses, a different treatment modality was selected as the reference category to perform the pairwise comparison ("no treatment" for Model 1, "surgery only" for Model 2, and "chemo only" for Model 3). The competing risk model was adjusted for age, race/ethnicity, marital status, histologic grade, AJCC T \& N category, molecular subtype, and treatment modality (data not shown).

to relatively favorable prognosis. Those with brain involvement were categorized as M1c. In our study, patients with Mlb disease had an approximately $40 \%$ increased mortality risk, and those with Mlc disease exhibited greater than 2-fold higher risk of mortality compared with those with Mla disease. Similar directions of the association were observed across different molecular subtypes.

Palliative systemic therapy is usually considered the therapeutic backbone for patients with metastatic breast cancer. ${ }^{12,14}$ Despite this, studies have reported that approximately $40 \%$ of women receive locoregional surgery and some patients desire a complete excision of primary tumor for psychosocial reasons, ${ }^{23-25}$ and many other reports reached an entirely different conclusion regarding primary tumor surgery versus systemic therapy. A Turkish study (MF07-01) reported a 34\% higher overall survival for patients receiving locoregional surgery combined with chemotherapy compared with those receiving chemotherapy 
alone (HR, 0.66; 95\% CI, 0.49-0.88; $P=.005$ ), especially in those with more indolent disease, such as HoR+/HER2subtype and single-bone involvement. ${ }^{15}$ Similarly, our data revealed that for patients with bone metastases (mainly classified as M1a), primary tumor surgery might provide a favorable prognosis. In addition, numerous retrospective studies and meta-analyses had reaffirmed the survival benefit associated with locoregional surgery with or without chemotherapy for advanced disease. ${ }^{23,24,26,27}$ On the contrary, an Indian trial reported a significant decrease in distant progressionfree survival (HR, 1.42; 95\% CI, $1.08-1.85 ; P=.012$ ) in patients receiving locoregional treatment compared with chemotherapy only. ${ }^{28}$ Cautious interpretation was suggested because of the insufficient use of subsequent systemic therapy and targeted anti-HER2 therapy. In addition, the lack of stratification factors might lead to unbalanced inclusion.

In our study, we attempted to account for margin status, which was not accessible in the SEER database, and divided the whole population into patients receiving no surgery, lumpectomy, or mastectomy. We hypothesized that patients undergoing mastectomy may be more likely to receive a negative margin, and thus surgery types might be an alternative indicator for margin status to some extent. ${ }^{29}$ Many previous works reported no survival gain from surgery with positive margins in metastatic setting. ${ }^{25,30,31}$ However, our study presented no survival difference between women receiving mastectomy or lumpectomy, and therefore it could be speculated that many patients may obtain clear margins after lumpectomy. Similarly, Lane et $\mathrm{a}^{25}$ reported a positive margin rate of $<20 \%$ for patients receiving lumpectomy or mastectomy based on a national database. However, we cannot determine whether more aggressive surgery (simple of modified mastectomy) can provide additional benefits.

We also found that surgical resection did not improve survival for women with TNBC across M1 subcategories. Similarly, Neuman et $\mathrm{a}^{32}$ reported that survival gain was observed among women with ER/PR-positive and/or HER2amplified disease, although not in those with TNBC, indicating that less benefit might be obtained from primary surgery of metastatic disease with aggressive tumor biology.

Our findings may have some implications for clinical practice. For patients with Mla disease, the combination of surgery and chemotherapy is preferred due to the approximately $50 \%$ reduction in mortality risk. It is reasonable to assume that these patients present better performance status and more favorable prognosis and that they can tolerate and benefit from the aggressive combination. For the Mlb subcategory, which consists of patients with different metastatic burden, combined treatment has no clinical necessity because only statistically borderline survival improvement is observed between monotherapies and combination therapy; both surgery alone and chemotherapy alone may benefit specific subgroups of patients. Further investigation is required. As for patients with Mlc disease, systemic chemotherapy alone may be considered after considering the surgical difficulty, potential decline in quality of life, possible visceral crisis, and palliation of symptoms. Among women who did not receive RT, those receiving chemotherapy alone experienced a $30 \%(70 \%$ in those with TNBC) reduction in mortality risk compared with those receiving surgery only. The fact that the lowest SHRs were associated with combined therapy may be attributed to selection bias, and we believe that this bias increases with upstaging of the metastatic disease. Women with M1c disease tend to present with organ failure and short life expectancy ${ }^{26}$; however, those receiving locoregional surgery may have better general well-being, thus leading to the lowest SHRs observed.

Despite the possible benefits from surgery, systemic therapy is still the preferred choice for MBC. ${ }^{12,14}$ In our study, the Mla subcategory contains more ER/PR-positive disease, which can be well-controlled by some state-of-theart therapies, such as the combination of CDK4/6 inhibitors with endocrine therapies. Nonetheless, it is of great interest and necessity to study whether and when to perform primary tumor surgery for selected patients with longterm disease control by CDK4/6 inhibitors.

This work has several limitations. First, some variables were not available in SEER, such as the number and size of metastatic lesions and other metastatic sites. Therefore, involvement of contralateral axillary lymph nodes, pleura, and other sites was not included for further subdivision of M1 category. The variable "number of metastatic sites," which was the total number of sites involved in the bone, brain, liver, or lung, might cause potential bias. Second, SEER did not provide detailed information on locoregional treatment, making it impossible to adjust for margin status, surgery of the axilla and metastatic disease, and use of RT. Neither could we account for the systemic therapy in terms of use, timing of initiation, and patient response, preventing adjustment for the sequence of surgery and systemic therapy and other potential confounders. Third, other confounders such as Charlson comorbidity score were not adjusted for, and the possibly unbalanced distribution of comorbidity might be another source of selection bias, resulting in the observed benefit from surgery. Finally, each Ml subcategory consists of a heterogeneous group of patients; therefore, the treatment advice based on M1 subcategory may not apply to each individual in that subcategory. We acknowledge that the Ml category subdivisions and the observed relationship between Ml subcategories and treatment modality should be prospectively validated. As treatment moves toward precision and personalization, 
multigene assays and biologic subtypes in addition to cancer staging may also play an increasingly important role in clinical decision-making.

\section{Conclusions}

The subdivision of Ml category reported herein, taking into account the metastatic sites and number of sites involved, may provide both patients with de novo MBC and oncologists with critical information for informing prognosis and help facilitate treatment choice. Treatment offered to women with metastatic disease should be determined by a coordinated, multidisciplinary team. As the prolongation of survival resulted from systemic therapy, primary tumor surgery may further benefit selected patients who respond well to systemic therapy. Although results of several related randomized trials are awaited, patients with de novo $\mathrm{MBC}$ should be provided with the currently available evidence when determining treatment approach.

Submitted November 3, 2018; accepted for publication June 18, 2019

Author contributions: Study concept and design: Lin, Wu, Zhu. Data acquisition: Lin, Ding, Goh. Data analysis and interpretation: Lin, Wu, Ding, Andriani, Lu, Shen. Manuscript preparation: All authors. Final approval: Zhu.

Disclosures: The authors have disclosed that they have not received any financial considerations from any person or organization to support the preparation, analysis, results, or discussion of this article.

Correspondence: Li Zhu, MD, PhD, Comprehensive Breast Health Center, Ruijin Hospital, 197 Ruijin Er Road, Huangpu District, Shanghai 200025, China. Email: zhuli8@yeah.net

\section{References}

1. DeSantis CE, Ma J, Goding Sauer A, et al. Breast cancer statistics, 2017, racial disparity in mortality by state. CA Cancer J Clin 2017;67:439-448.

2. Mariotto $A B$, Etzioni $R$, Hurlbert $M$, et al. Estimation of the number of women living with metastatic breast cancer in the United States. Cancer Epidemiol Biomarkers Prev 2017;26:809-815

3. Largillier R, Ferrero JM, Doyen J, et al. Prognostic factors in 1,038 women with metastatic breast cancer. Ann Oncol 2008;19:2012-2019.

4. Hölzel D, Eckel R, Bauerfeind I, et al. Survival of de novo stage IV breast cancer patients over three decades. J Cancer Res Clin Oncol 2017;143. 509-519.

5. Andre F, Slimane K, Bachelot $T$, et al. Breast cancer with synchronous metastases: trends in survival during a 14-year period. J Clin Oncol 2004; 22:3302-3308.

6. Lobbezoo DJ, van Kampen RJ, Voogd AC, et al. Prognosis of metastatic breast cancer: are there differences between patients with de novo and recurrent metastatic breast cancer? Br J Cancer 2015;112:1445-1451.

7. Amin MB, Edge SB, Greene FL, et al. AJCC Cancer Staging Manual, 8th ed. New York, NY: Springer; 2017.

8. Brierley JD, Gospodarowicz MK, Wittekind C, et al. TNM classification of malignant tumours, 8th ed. Oxford, UK: Wiley Blackwell; 2017.

9. Edge SB, Compton CC. The American Joint Committee on Cancer: the 7th Edition of the AJCC Cancer Staging Manual and the Future of TNM. Ann Surg Oncol 2010;17:1471-1474.

10. Zou X, You R, Liu H, et al. Establishment and validation of M1 stage subdivisions for de novo metastatic nasopharyngeal carcinoma to better predict prognosis and guide treatment. Eur J Cancer 2017; 77(Supplement C):117-126.

11. Shen L, Li W, Wang S, et al. Image-based multilevel subdivision of M1 category in TNM Staging System for metastatic nasopharyngeal carcinoma. Radiology 2016;280:805-814.

12. Harbeck N, Gnant M. Breast cancer. Lancet 2017;389:1134-1150.

13. Gradishar WJ, Anderson BO, Aft R, et al. NCCN Clinical Practice Guidelines in Oncology: Breast Cancer. Version 1.2018. To view the most recent version, visit NCCN.org. Accessed March 20, 2018.

14. Cardoso F, Costa A, Senkus E, et al. 3rd ESO-ESMO International Consensus Guidelines for Advanced Breast Cancer (ABC 3). Ann Oncol 2017;28:16-33.

15. Soran A, Ozmen V, Ozbas $S$, et al. Randomized trial comparing resection of primary tumor with no surgery in stage IV breast cancer at presentation: protocol MF07-01. Ann Surg Oncol 2018;25:3141-3149.

16. Surveillance, Epidemiology, and End Results Program. SEER*Stat Database: Incidence - SEER 9 Regs Research Data, Nov 2017 Sub (1973-2015) < Katrina/Rita Population Adjustment $>$ - Linked To County Attributes - Total U.S., 1969-2016 Counties, National Cancer Institute, DCCPS, Surveillance Research Program. Based on November 2017 submission. Released April 2018.
17. Gray RJ. A class of K-sample tests for comparing the cumulative incidence of a competing risk. Ann Stat 1988;16:1141-1154.

18. Fine JP, Gray RJ. A proportional hazards model for the subdistribution of a competing risk. J Am Stat Assoc 1999;94:496-509.

19. Waks AG, Winer EP. Breast cancer treatment: a review. JAMA 2019, 321:288-300

20. Rahman ZU, Frye DK, Smith TL, et al. Results and long term follow-up for 1581 patients with metastatic breast carcinoma treated with standard dose doxorubicin-containing chemotherapy: a reference. Cancer 1999;85:104-111.

21. Yamamura J, Kamigaki S, Fujita J, et al. The difference in prognostic outcomes between De Novo stage IV and recurrent metastatic patients with hormone receptor-positive, HER2-negative breast cancer. In Vivo 2018;32:353-358

22. Cummings MC, Simpson PT, Reid LE, et al. Metastatic progression of breast cancer: insights from 50 years of autopsies. J Pathol 2014;232:23-31.

23. Thomas A, Khan SA, Chrischilles EA, et al. Initial surgery and survival in stage IV breast cancer in the United States, 1988-2011. JAMA Surg 2016;151:424-431.

24. Warschkow R, Güller U, Tarantino I, et al. Improved survival after primary tumor surgery in metastatic breast cancer: a propensity-adjusted, population-based SEER trend analysis. Ann Surg 2016;263:1188-1198.

25. Lane WO, Thomas SM, Blitzblau RC, et al. Surgical resection of the primary tumor in women with de novo stage IV breast cancer: contemporary practice patterns and survival analysis. Ann Surg 2019;269:537-544.

26. Lu S, Wu J, Fang Y, et al. The impact of surgical excision of the primary tumor in stage IV breast cancer on survival: a meta-analysis. Oncotarget 2017;9:11816-11823

27. Petrelli F, Barni S. Surgery of primary tumors in stage IV breast cancer: an updated meta-analysis of published studies with meta-regression. Med Oncol 2012;29:3282-3290.

28. Badwe R, Hawaldar R, Nair N, et al. Locoregional treatment versus no treatment of the primary tumour in metastatic breast cancer: an openlabel randomised controlled trial. Lancet Oncol 2015;16:1380-1388.

29. Ruiterkamp J, Ernst MF, van de Poll-Franse LV, et al. Surgical resection of the primary tumour is associated with improved survival in patients with distant metastatic breast cancer at diagnosis. Eur J Surg Oncol 2009;35:1146-1151.

30. Khan SA, Stewart AK, Morrow M. Does aggressive local therapy improve survival in metastatic breast cancer? Surgery 2002;132:620-626; discussion 626-627.

31. Rapiti E, Verkooijen HM, Vlastos G, et al. Complete excision of primary breast tumor improves survival of patients with metastatic breast cancer at diagnosis. J Clin Oncol 2006;24:2743-2749.

32. Neuman HB, Morrogh M, Gonen M, et al. Stage IV breast cancer in the era of targeted therapy: does surgery of the primary tumor matter? Cancer 2010;116:1226-1233. 
Supplemental online content for:

\section{Subdivision of M1 Stage for De Novo Metastatic Breast Cancer to Better Predict Prognosis and Response to Primary Tumor Surgery}

Caijin Lin, M.Med; Jiayi Wu, MD; Shuning Ding, M.Med; Chihwan Goh, M.Med; Lisa Andriani, M.Med; Shuangshuang Lu, MD; Kunwei Shen, MD, PhD; and Li Zhu, MD, PhD

J Natl Compr Canc Netw 2019;17(12):1521-1528

eFigure 1: Cumulative Incidence Curves of Cancer-Specific Mortality Stratified by Site

eFigure 2: Cumulative Incidence Curves of Cancer-Specific Mortality and All-Cause Mortality

eFigure 3: Cumulative Incidence Curves of Cancer-Specific Mortality Stratified by Treatment Modality

eTable 1: Detailed Baseline Characteristics

eTable 2: Baseline Characteristics Stratified by M1 Subcategory

eTable 3: Univariate and Multivariate Analysis of Cancer-Specific Mortality in the Training Set: A Competing Risk Regression Model

eTable 4: Description of Different Patient Groups Based on Independent Prognostic Factors

eTable 5: Multivariate Analysis of Cancer-Specific Mortality: A Competing Risk Model

eTable 6: Multivariate Analysis of All-Cause Mortality: Cox Proportional Hazards Model

eTable 7: Baseline Patient Characteristics by Molecular Subtype

eTable 8: Median Survival by M1 Subcategory, Stratified by Molecular Subtype and Histologic Grade

eTable 9: Multivariate Analysis of Cancer-Specific Mortality by Treatment Modality

eTable 10: Association of Primary Tumor Surgery With Mortality

eAppendix 1: Validation of M1 Category Subdivision System 
A

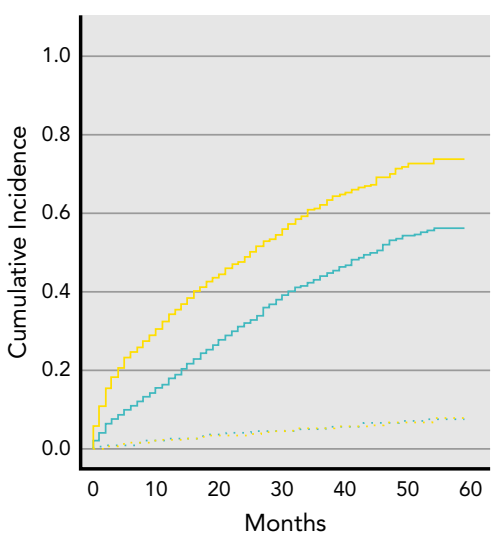

D

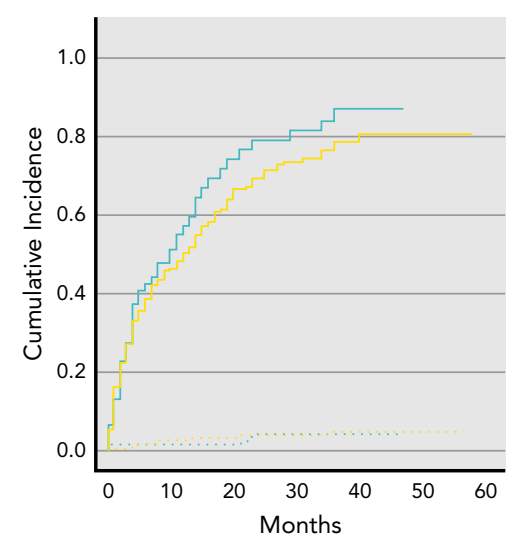

B

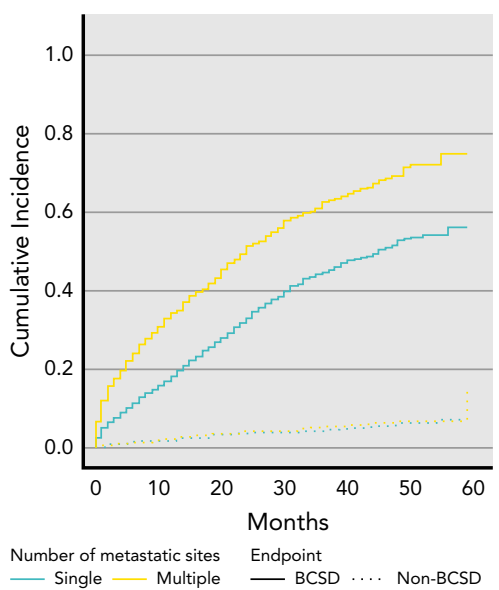

E

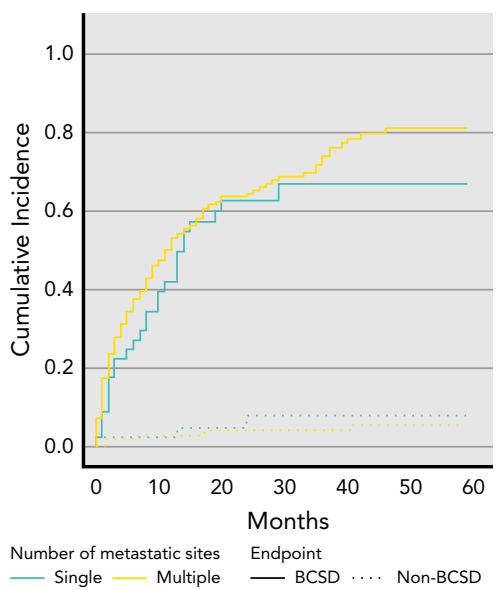

C

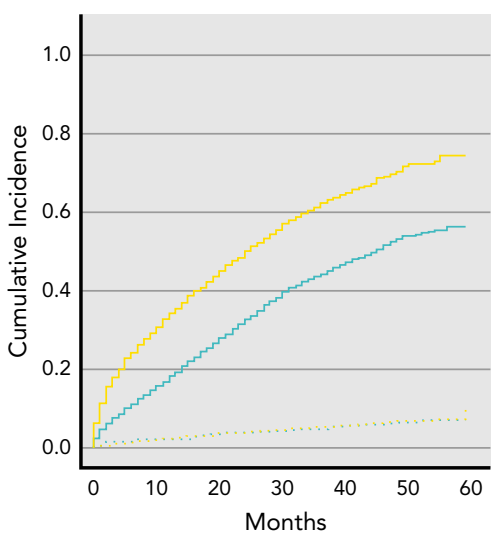

$\mathbf{F}$

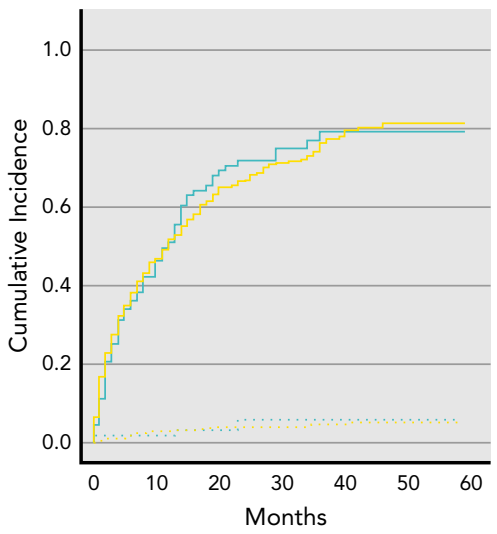

eFigure 1. Cumulative incidence curves of cancer-specific mortality stratified by single/multiple sites involved in the (A-C) absence or $(D-F)$ presence of brain involvement in the $(A, D)$ training, $(B, E)$ validation, and $(C, F)$ whole sets.

Abbreviation: BCSD, breast cancer-specific death. 
A

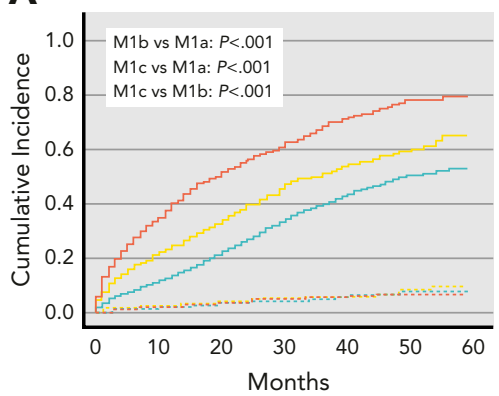

D

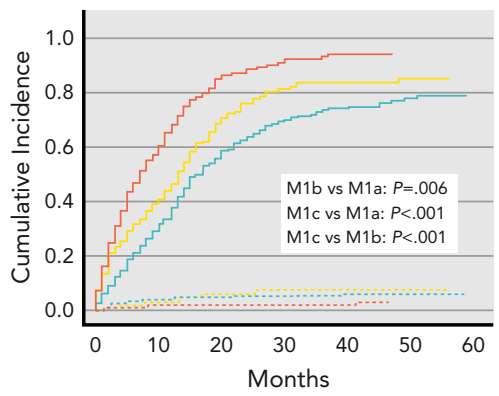

G

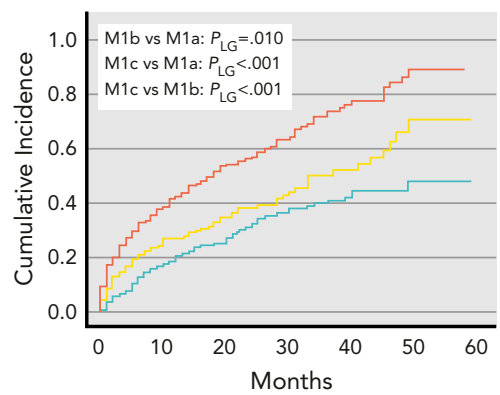

B

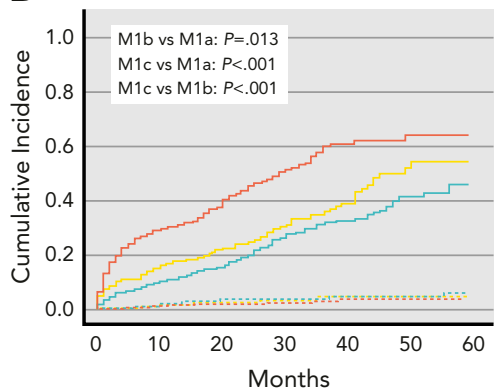

E

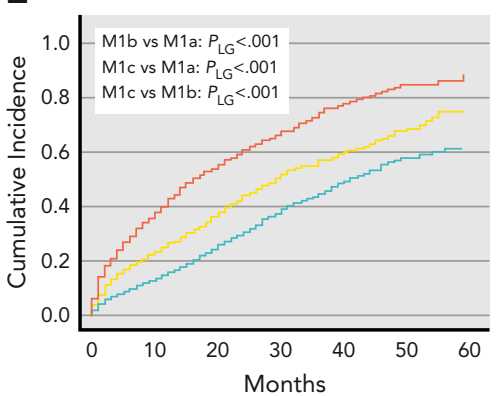

H

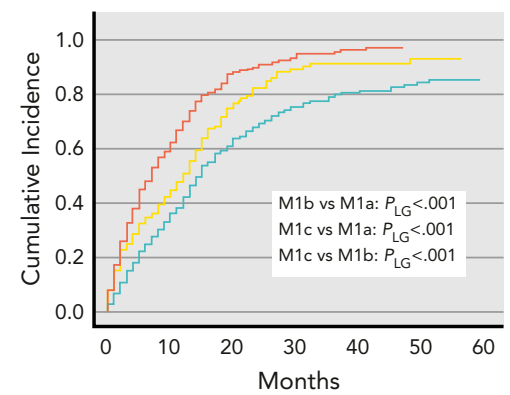

C

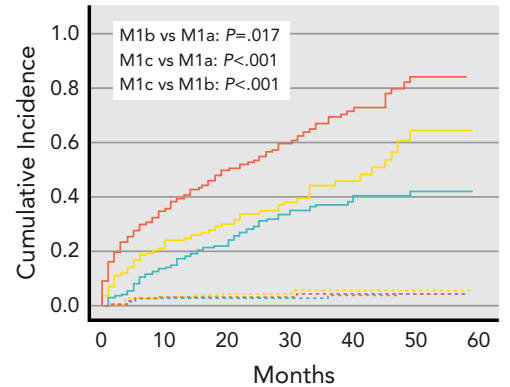

F

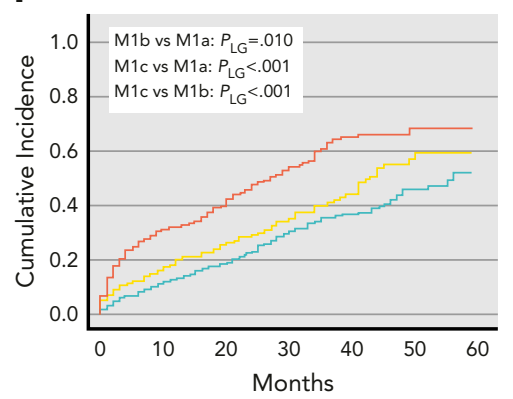

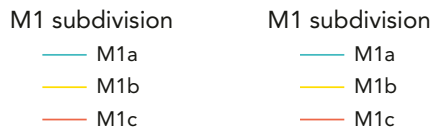

Endpoint

BCSD

...... Non-BCSD

eFigure 2. Cumulative incidence curves of (A-D) cancer-specific and (E-H) all-cause mortality stratified by $M 1$ subcategories in patients with (A, $E)$ HoR+/HER2-, (B, F) HoR+/HER2+, (C, G) HoR-/HER2+, or (D, H) triple-negative breast cancer in the whole set. $P$ values were estimated and compared based on Gray's test (A-D) or log-rank test (E-H).

Abbreviations: BCSD, breast cancer-specific death; HoR, hormone receptor; LG, log-rank. 
A

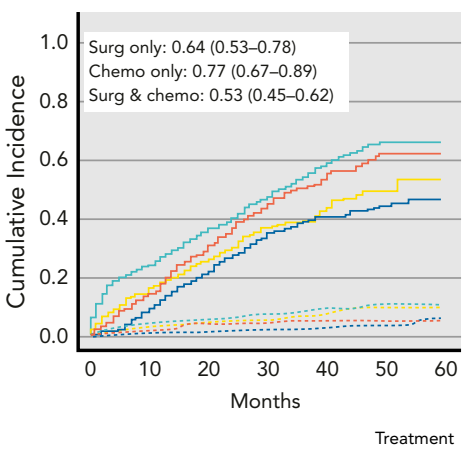

D

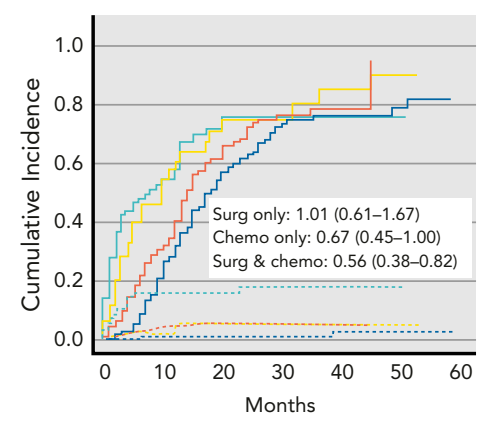

B

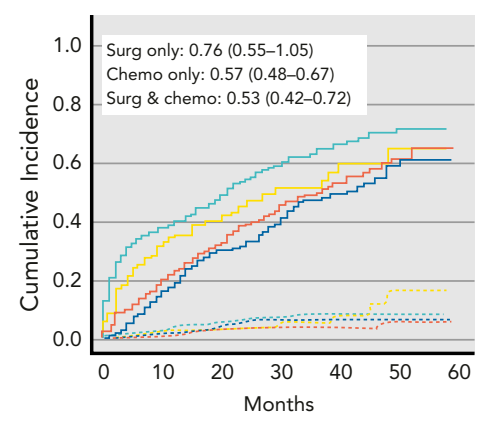

Surg only Chemo only — Surg \& chemo $\stackrel{\text { Endpoint }}{\text { B BCSD }}$

E

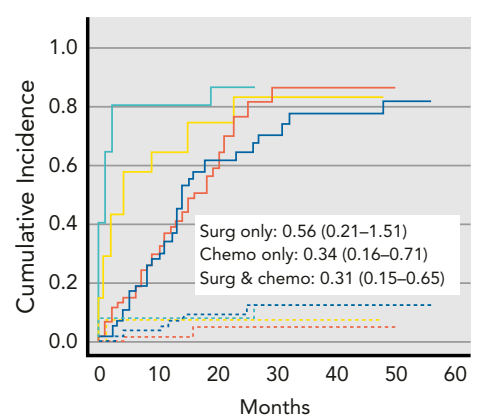

C

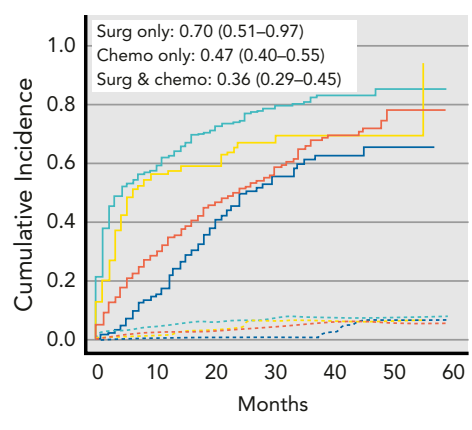

Non-BCSD

Treatment Endpoint

$\mathbf{F}$

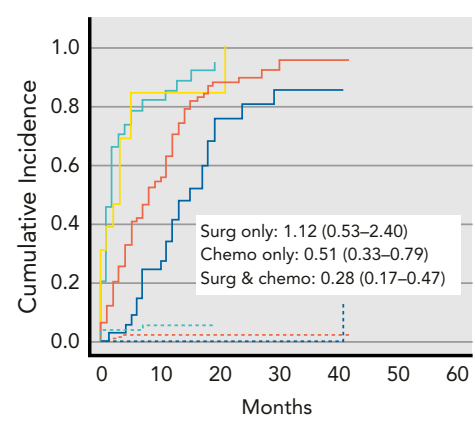

eFigure 3. Cumulative incidence curves of cancer-specific mortality stratified by treatment modality across ( $A, D) M 1 a,(B, E) M 1 b$, and $(C, F)$ M1c in patients not receiving RT (A-C) and those with TNBC not receiving RT (D-F). Patients receiving no treatment were used as the reference category and data are presented as SHR $(95 \% \mathrm{Cl})$.

Abbreviations: BCSD, breast cancer-specific death; chemo, chemotherapy; RT, radiotherapy; SHR, subdistribution hazard ratio; surg, surgery. 


\section{eTable 1. Detailed Baseline Patient Characteristics $(\mathbf{N}=\mathbf{8 , 5 8 2})$}

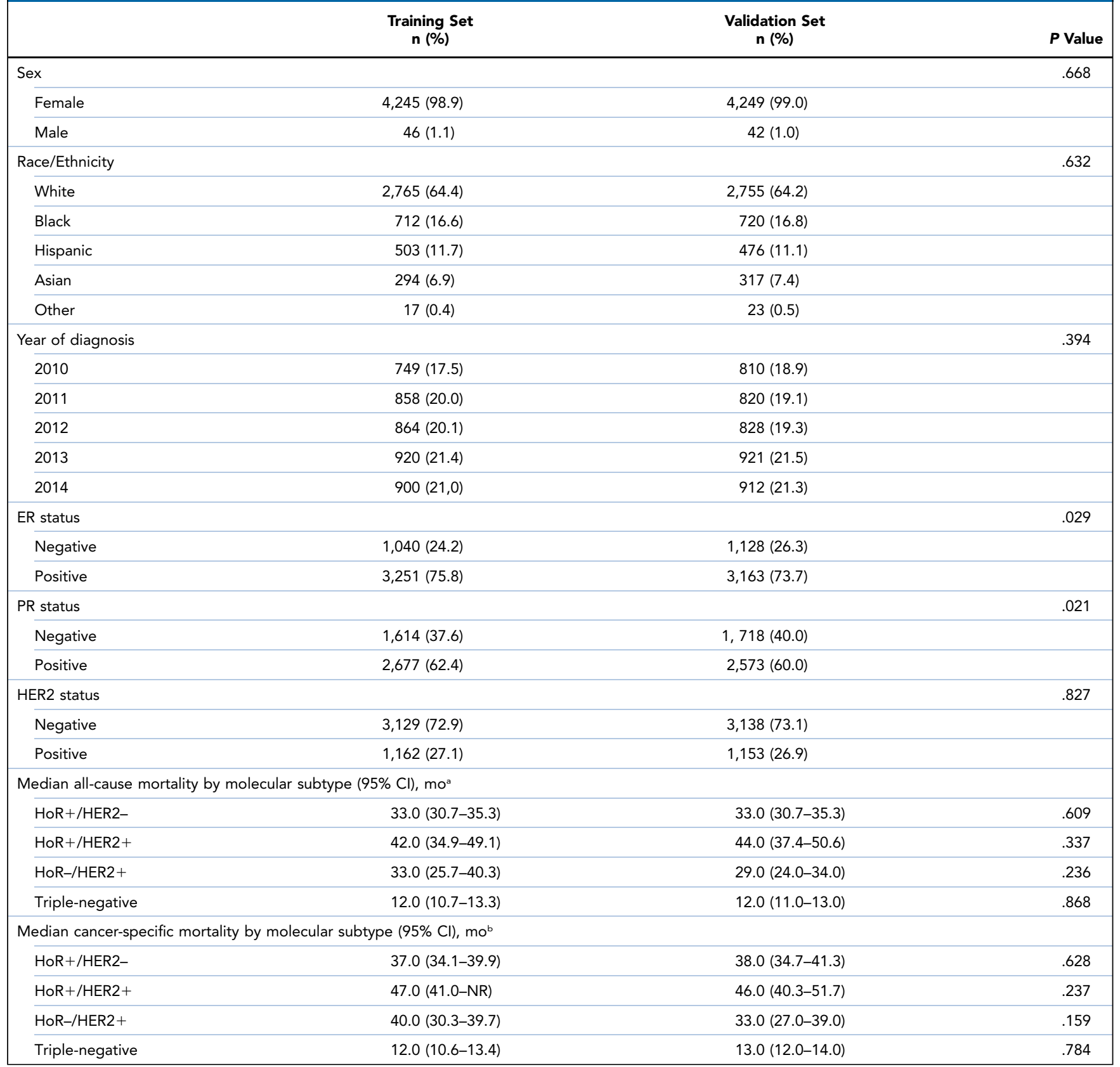

Abbreviations: ER, estrogen receptor; HoR, hormone receptor; NR, not reached; PR, progesterone receptor.

a $P$ values estimated and compared using log-rank test.

bP values estimated and compared using Gray's test. 


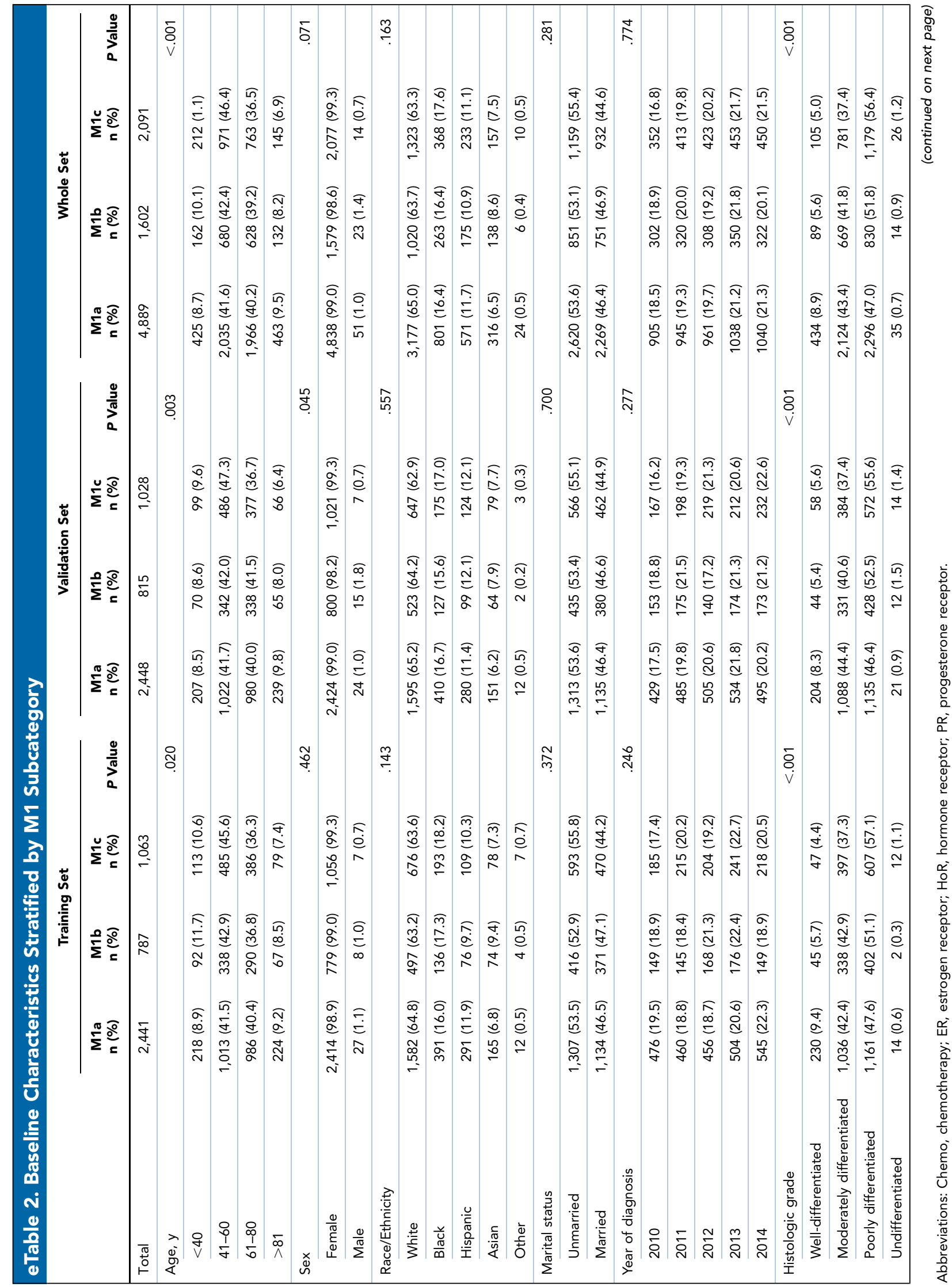




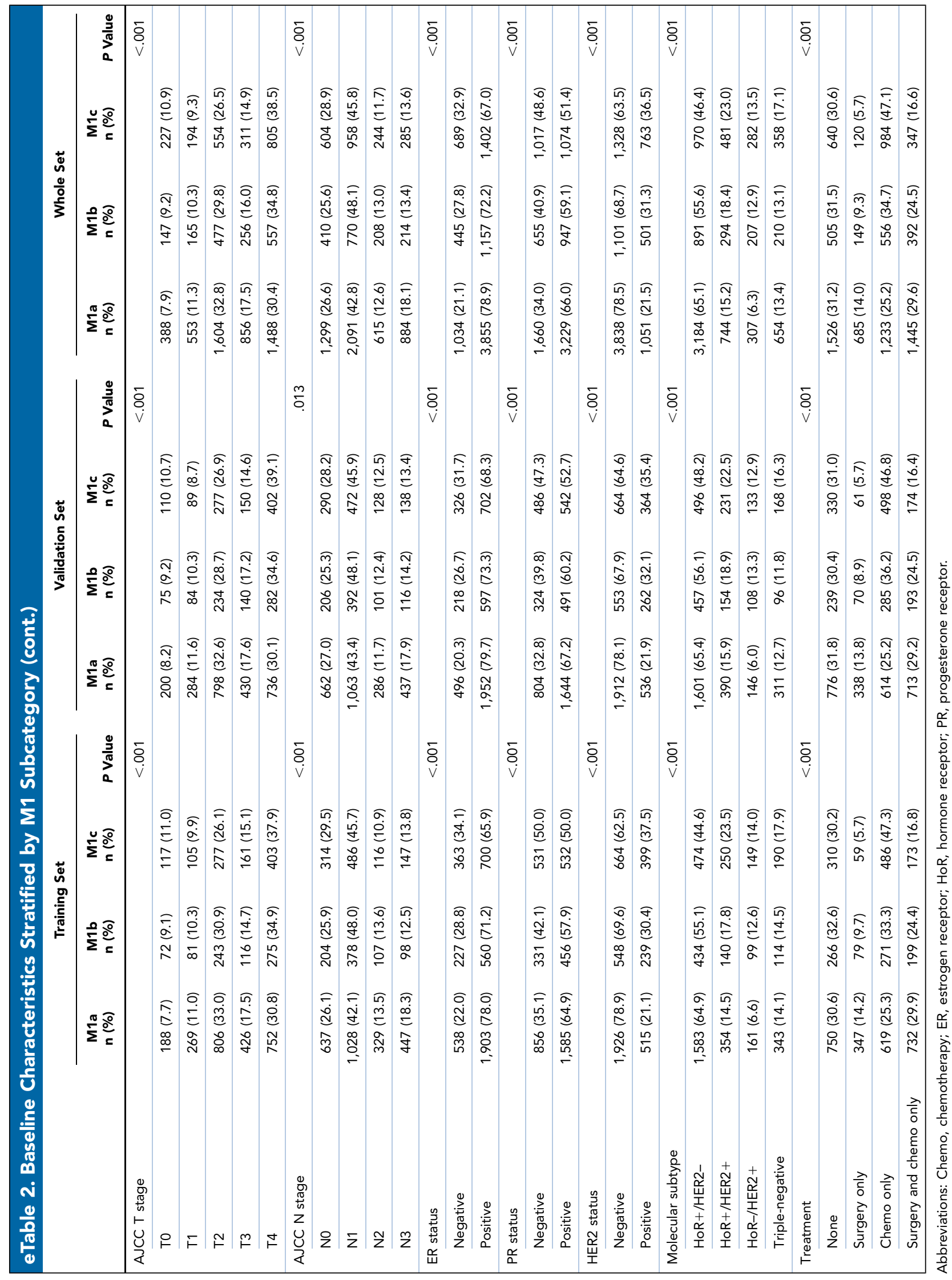


eTable 3. Univariate and Multivariate Analysis of Cancer-Specific Mortality in the Training Set: A Competing Risk Regression Model

\begin{tabular}{|c|c|c|c|c|}
\hline & \multicolumn{2}{|c|}{ Univariate Analysis } & \multicolumn{2}{|c|}{ Multivariate Analysis } \\
\hline \multicolumn{5}{|l|}{ Age, y } \\
\hline$<40$ & Ref & & Ref & \\
\hline $41-60$ & $1.38(1.15-1.65)$ & .001 & $1.35(1.12-1.62)$ & .002 \\
\hline $61-80$ & $1.69(1.42-2.03)$ & $<.001$ & $1.56(1.29-1.89)$ & $<.001$ \\
\hline \multicolumn{5}{|l|}{ Sex } \\
\hline Female & Ref & & - & - \\
\hline Male & $0.85(0.51-1.44)$ & .547 & - & - \\
\hline \multicolumn{5}{|l|}{ Race/Ethnicity } \\
\hline White & Ref & & Ref & \\
\hline Other & $0.62(0.29-1.32)$ & .215 & $0.52(0.19-1.42)$ & .203 \\
\hline \multicolumn{5}{|l|}{ Marital status } \\
\hline Unmarried & Ref & & Ref & \\
\hline Married & $0.75(0.69-0.83)$ & $<.001$ & $0.88(0.79-0.98)$ & .015 \\
\hline \multicolumn{5}{|l|}{ Histologic grade } \\
\hline Well-differentiated & Ref & & Ref & \\
\hline Moderately differentiated & $1.27(1.03-1.55)$ & .024 & $1.25(1.01-1.55)$ & .042 \\
\hline Poorly differentiated & $1.89(1.55-2.31)$ & $<.001$ & $1.73(1.39-2.14)$ & $<.001$ \\
\hline Undifferentiated & $1.82(0.99-3.33)$ & .054 & $1.61(0.94-2.75)$ & .081 \\
\hline \multicolumn{5}{|l|}{ AJCC N stage } \\
\hline NO & Ref & & Ref & \\
\hline $\mathrm{N} 1$ & $0.87(0.77-0.99)$ & .033 & $0.84(0.74-0.96)$ & .010 \\
\hline N2 & $0.82(0.70-0.96)$ & .017 & $0.88(0.74-1.04)$ & .123 \\
\hline N3 & $0.79(0.67-0.92)$ & .003 & $0.81(0.69-0.96)$ & .014 \\
\hline \multicolumn{5}{|l|}{ Molecular subtype } \\
\hline HoR+/HER2- & Ref & & Ref & \\
\hline HoR +/HER2+ & $0.79(0.68-0.91)$ & .001 & $0.76(0.65-0.89)$ & $<.001$ \\
\hline HoR-/HER2+ & $1.06(0.89-1.26)$ & .517 & $0.94(0.78-1.14)$ & .533 \\
\hline Triple-negative & $2.62(2.34-2.94)$ & $<.001$ & $2.42(2.10-2.79)$ & $<.001$ \\
\hline \multicolumn{5}{|l|}{ Bone involvement } \\
\hline No & Ref & & - & - \\
\hline Yes & $0.92(0.84-1.02)$ & .124 & - & - \\
\hline
\end{tabular}

Abbreviations: Chemo, chemotherapy; ER, estrogen receptor; HoR, hormone receptor; PR, progesterone receptor. 
eTable 3. Univariate and Multivariate Analysis of Cancer-Specific Mortality in the Training Set: A Competing Risk Regression Model (cont.)

\begin{tabular}{|c|c|c|c|c|}
\hline & \multicolumn{2}{|c|}{ Univariate Analysis } & \multicolumn{2}{|c|}{ Multivariate Analysis } \\
\hline \multicolumn{5}{|l|}{ Brain involvement } \\
\hline No & Ref & & Ref & \\
\hline Yes & $2.51(2.16-2.92)$ & $<.001$ & $2.13(1.80-2.52)$ & $<.001$ \\
\hline \multicolumn{5}{|l|}{ Liver involvement } \\
\hline Yes & $1.79(1.62-1.98)$ & $<.001$ & $1.71(1.51-1.95)$ & $<.001$ \\
\hline \multicolumn{5}{|l|}{ Lung involvement } \\
\hline No & Ref & & Ref & \\
\hline Yes & $1.57(1.43-1.73)$ & $<.001$ & $1.14(1.00-1.28)$ & .043 \\
\hline \multicolumn{5}{|c|}{ Number of metastatic sites } \\
\hline \multicolumn{5}{|l|}{ Binary classifications 1} \\
\hline \multicolumn{5}{|l|}{ Binary classifications 2} \\
\hline 1 or 2 sites & Ref & & Ref & \\
\hline$>2$ sites & $2.33(2.03-2.69)$ & $<.001$ & $1.11(0.91-1.35)$ & .318 \\
\hline \multicolumn{5}{|l|}{ Treatment modality } \\
\hline None & Ref & & Ref & \\
\hline Surgery only & $0.63(0.53-0.74)$ & $<.001$ & $0.67(0.56-0.80)$ & $<.001$ \\
\hline Chemo only & $0.74(0.66-0.82)$ & $<.001$ & $0.59(0.52-0.68)$ & $<.001$ \\
\hline Surgery and chemo & $0.48(0.42-0.54)$ & $<.001$ & $0.44(0.38-0.52)$ & $<.001$ \\
\hline
\end{tabular}

Abbreviations: Chemo, chemotherapy; ER, estrogen receptor; HoR, hormone receptor; PR, progesterone receptor.

\section{eTable 4. Description of Different Patient Groups Based on Independent Prognostic Factors}

\begin{tabular}{|c|c|c|c|c|c|c|}
\hline Brain Involvement & Liver Involvement & Multiple Sites Involvement & Group & M1 Subcategory & SHR $(95 \% \mathrm{Cl})$ & $P$ value \\
\hline Yes & No & Yes & $A^{a}$ & M1c & & - \\
\hline No & Yes & Yes & $\mathrm{B}^{\mathrm{b}}$ & M1c & \multirow{2}{*}{$\begin{array}{l}\text { Group B vs C } \\
\quad 1.61(1.33-1.94)\end{array}$} & \multirow[b]{2}{*}{$<.001$} \\
\hline No & Yes & No & $C^{c}$ & M1b & & \\
\hline & No & Yes & $D^{d}$ & M1b & $\begin{array}{l}\text { Group D vs E } \\
1.40(1.19-1.64)\end{array}$ & $<.001$ \\
\hline No & No & No & $\mathrm{E}^{\mathrm{e}}$ & M1a & - & - \\
\hline
\end{tabular}

Abbreviation: SHR, subdistribution hazard ratio.

alnvolvement of brain, irrespective of number of metastatic sites.

bInvolvement of liver and multiple sites except brain.

'Liver involvement only.

Involvement of multiple sites except brain or liver.

eInvolvement of single site except brain or liver. 


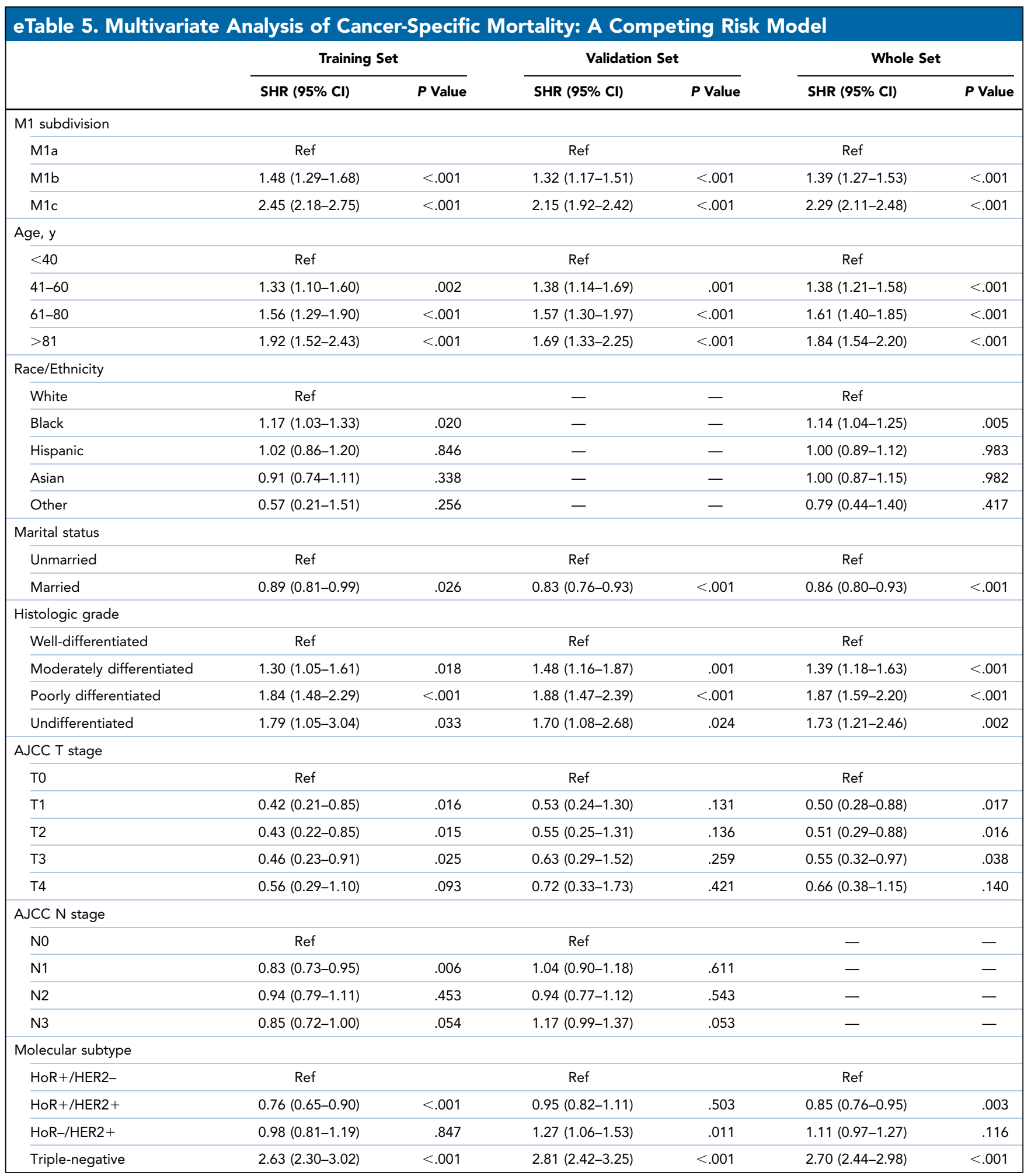

Abbreviations: Chemo, chemotherapy; HoR, hormone receptor; SHR, subdistribution hazard ratio. 
10 - Lin et al

\section{eTable 5. Multivariate Analysis of Cancer-Specific Mortality: A Competing Risk Model (cont.)}

Training Set

Validation Set

Whole Set

SHR $(95 \% \mathrm{Cl}) \quad P$ Value

SHR $(95 \% \mathrm{Cl}) \quad P$ Value

SHR $(95 \% \mathrm{Cl})$

$P$ Value

Treatment modality

None

$$
\text { Ref }
$$

Ref

Ref

Surgery only

$0.66(0.55-0.79)$

$0.59(0.52-0.68)$

$<.001$

$0.52(0.44-0.63)$

$0.60(0.53-0.69)$

$<.001$

$0.59(0.52-0.68)$

$0.60(0.54-0.66)$

$<.001$

Surgery and chemo

$0.44(0.38-0.51)$

$<.001$

$0.32(0.28-0.38)$

$<.001 \quad 0.38(0.35-0.43)$

$<.001$

Abbreviations: Chemo, chemotherapy; HoR, hormone receptor; SHR, subdistribution hazard ratio. 
eTable 6. Multivariate Analysis of All-Cause Mortality: Cox Proportional Hazards Model

\begin{tabular}{|c|c|c|c|c|c|c|}
\hline & \multicolumn{2}{|c|}{ Training Set } & \multicolumn{2}{|c|}{ Validation Set } & \multicolumn{2}{|c|}{ Whole Set } \\
\hline \multicolumn{7}{|l|}{ M1 subdivision } \\
\hline M1a & Ref & & Ref & & Ref & \\
\hline M1b & $1.47(1.30-1.66)$ & $<.001$ & $1.35(1.20-1.53)$ & $<.001$ & $1.41(1.29-1.53)$ & $<.001$ \\
\hline M1c & $2.38(2.14-2.65)$ & $<.001$ & $2.09(1.88-2.33)$ & $<.001$ & $2.22(2.06-2.41)$ & $<.001$ \\
\hline$<40$ & Ref & & Ref & & Ref & \\
\hline $41-60$ & $1.33(1.10-1.60)$ & .003 & $1.32(1.08-1.62)$ & .002 & $1.34(1.17-1.54)$ & $<.001$ \\
\hline $61-80$ & $1.71(1.41-2.07)$ & $<.001$ & $1.64(1.34-2.01)$ & $<.001$ & $1.71(1.49-1.96)$ & $<.001$ \\
\hline$>81$ & $2.38(1.90-2.98)$ & $<.001$ & $2.25(1.78-2.84)$ & $<.001$ & $2.33(1.98-2.74)$ & $<.001$ \\
\hline \multicolumn{7}{|l|}{ Race/Ethnicity } \\
\hline Asian & $0.98(0.82-1.19)$ & .861 & - & - & $1.06(0.93-1.2)$ & .407 \\
\hline Other & $0.49(0.22-1.09)$ & .080 & - & - & $0.83(0.52-1.35)$ & .455 \\
\hline \multicolumn{7}{|l|}{ Marital status } \\
\hline Unmarried & Ref & & Ref & & Ref & \\
\hline Married & $0.86(0.78-0.95)$ & .002 & $0.82(0.74-0.90)$ & .026 & $0.84(0.79-0.9)$ & $<.001$ \\
\hline \multicolumn{7}{|l|}{ Histologic grade } \\
\hline Well-differentiated & Ref & & Ref & & Ref & \\
\hline Moderate differentiated & $1.29(1.05-1.57)$ & .014 & $1.32(1.07-1.63)$ & .018 & $1.31(1.13-1.51)$ & $<.001$ \\
\hline Poorly differentiated & $1.86(1.52-2.28)$ & $<.001$ & $1.71(1.38-2.11)$ & $<.001$ & $1.78(1.54-2.06)$ & $<.001$ \\
\hline $\mathrm{T} 4$ & $0.66(0.30-1.48)$ & .314 & $0.87(0.39-1.95)$ & .093 & $0.79(0.45-1.39)$ & .411 \\
\hline \multicolumn{7}{|l|}{ AJCC N stage } \\
\hline No & Ref & & - & - & Ref & \\
\hline N1 & $0.81(0.72-0.91)$ & $<.001$ & - & - & $0.89(0.82-0.97)$ & .010 \\
\hline N2 & $0.94(0.79-1.11)$ & .437 & - & - & $0.94(0.83-1.05)$ & .274 \\
\hline N3 & $0.79(0.67-0.93)$ & .004 & - & - & $0.95(0.85-1.06)$ & .358 \\
\hline \multicolumn{7}{|l|}{ Molecular subtype } \\
\hline HoR+/HER2- & Ref & & Ref & & Ref & \\
\hline HoR +/HER2+ & $0.79(0.68-0.91)$ & .001 & $0.94(0.82-1.09)$ & $<.001$ & $0.86(0.78-0.95)$ & .003 \\
\hline HoR-/HER2+ & $1.08(0.90-1.28)$ & .412 & $1.35(1.13-1.60)$ & .847 & $1.20(1.06-1.35)$ & .004 \\
\hline Triple-negative & $2.79(2.46-3.17)$ & $<.001$ & $3.16(2.76-3.62)$ & $<.001$ & $2.91(2.66-3.19)$ & $<.001$ \\
\hline \multicolumn{7}{|l|}{ Treatment modality } \\
\hline None & Ref & & Ref & & Ref & \\
\hline Surgery only & $0.67(0.57-0.78)$ & $<.001$ & $0.48(0.41-0.56)$ & $<.001$ & $0.57(0.51-0.64)$ & $<.001$ \\
\hline Chemo only & $0.55(0.49-0.62)$ & $<.001$ & $0.58(0.52-0.66)$ & $<.001$ & $0.57(0.53-0.62)$ & $<.001$ \\
\hline Surgery and chemo & $0.38(0.33-0.44)$ & $<.001$ & $0.29(0.25-0.34)$ & $<.001$ & $0.34(0.30-0.37)$ & $<.001$ \\
\hline
\end{tabular}

Abbreviations: chemo, chemotherapy; HoR, hormone receptor; HR, hazard ratio. 
eTable 7. Baseline Patient Characteristics by Molecular Subtype

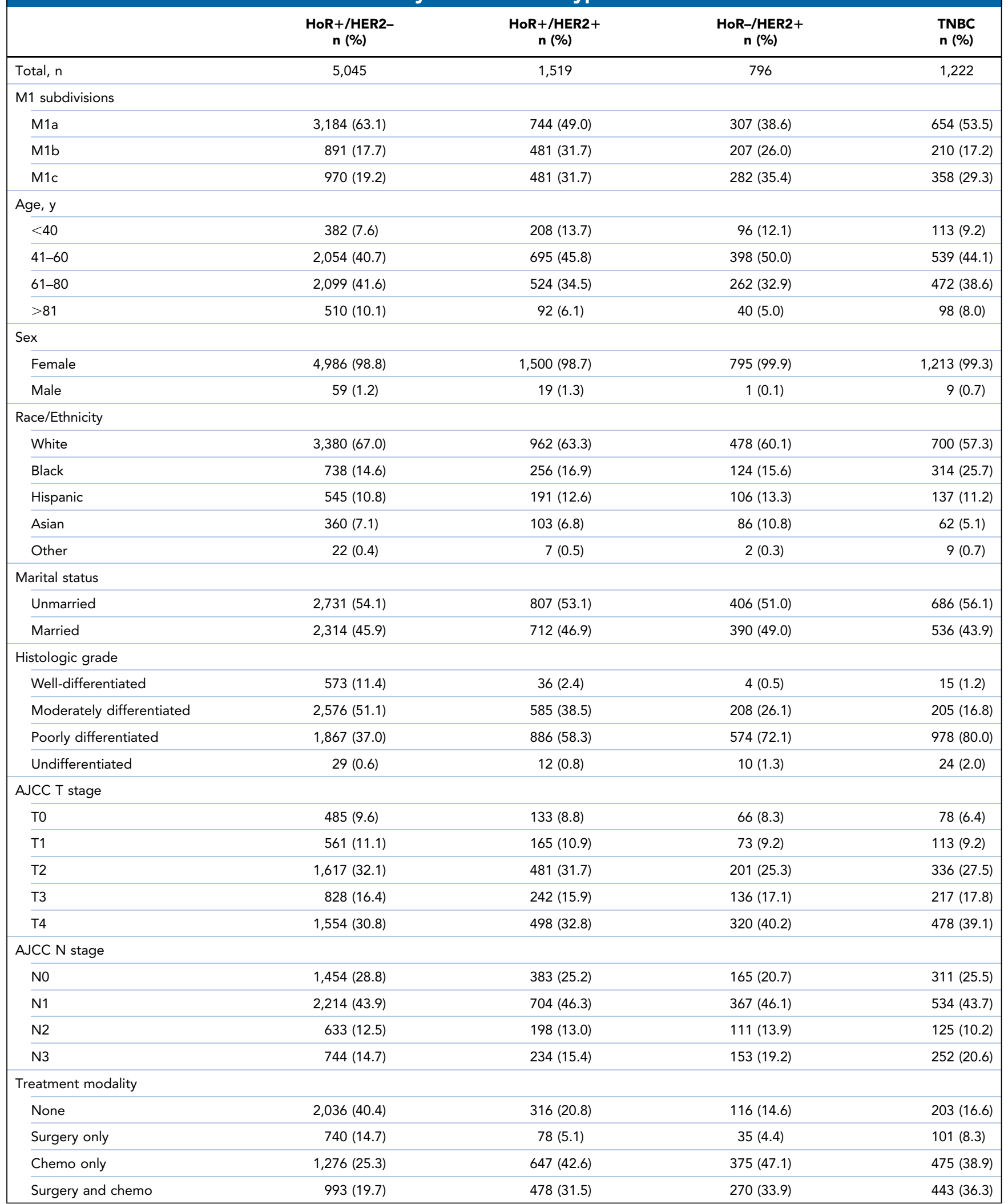

Abbreviation: chemo, chemotherapy; HoR, hormone receptor. 


\begin{tabular}{|c|c|c|c|c|c|c|c|}
\hline Molecular Subtype & Histologic Grade & M1 Subcategory & n (\%) & Median (IQR), mo & $P$ Value & Median (IQR), mo & $P$ Value \\
\hline \multirow[t]{8}{*}{ HoR+/HER2- } & \multirow[t]{3}{*}{ G1 } & M1a & $405(4.7)$ & NR (29.0-NR) & $<.001$ & 49.0 (26.0-NR) & $<.001$ \\
\hline & & M1b & $80(0.9)$ & 45.0 (30.0-NR) & & 45.0 (23.0-NR) & \\
\hline & & M1c & $88(1.0)$ & $36.0(11.0-49.0)$ & & $33.0(8.0-49.0)$ & \\
\hline & \multirow{2}{*}{$\mathrm{G} 2$} & M1b & $467(5.4)$ & 36.0 (15.0-NR) & & 31.0 (13.0-NR) & \\
\hline & & M1c & $471(5.5)$ & $21.0(5.0-39.0)$ & & $20.0(4.0-37.0)$ & \\
\hline & \multirow[t]{3}{*}{ G3 } & M1a & $1,141(13.3)$ & 35.0 (17.0-NR) & $<.001$ & 32.0 (16.0-NR) & $<.001$ \\
\hline & & M1b & $344(4.0)$ & 26.0 (10.0-NR) & & $23.0(9.0-47.0)$ & \\
\hline & & M1c & $411(4.8)$ & $14.0(5.0-36.0)$ & & $14.0(5.0-33.0)$ & \\
\hline \multirow{6}{*}{ HoR+/HER2+ } & \multirow[t]{3}{*}{$\mathrm{G} 2$} & M1a & $304(3.5)$ & NR (28.0-NR) & $<.001$ & 52.0 (25.0-NR) & $<.001$ \\
\hline & & M1b & $106(1.2)$ & 43.0 (28.0-NR) & & 42.0 (27.0-NR) & \\
\hline & & M1c & $175(2.0)$ & 21.0 (4.0-NR) & & 20.0 (4.0-NR) & \\
\hline & \multirow[t]{3}{*}{ G3 } & M1a & $423(4.9)$ & NR (28.0-NR) & $<.001$ & 55.0 (26.0-NR) & $<.001$ \\
\hline & & M1b & $181(2.1)$ & 49.0 (20.0-NR) & & 44.0 (16.0-NR) & \\
\hline & & M1c & $294(3.4)$ & 32.0 (8.0-NR) & & 30.0 (6.0-NR) & \\
\hline \multirow[t]{4}{*}{ HoR-/HER2+ } & \multirow[t]{3}{*}{ G1 } & M1a & $3(0.0)$ & $-^{a}$ & $-^{a}$ & - $^{\mathrm{a}}$ & $-^{a}$ \\
\hline & & M1b & $0(0.0)$ & $-^{a}$ & & $-^{a}$ & \\
\hline & & M1c & $1(0.0)$ & $一^{a}$ & & $一^{\mathrm{a}}$ & \\
\hline & $\mathrm{G} 2$ & M1a & $74(0.9)$ & NR (24.0-NR) & $<.001$ & NR (24.0-NR) & $<.001$ \\
\hline \multirow{8}{*}{ Triple-negative } & \multirow{2}{*}{ G1 } & M1b & $2(0.0)$ & $7.0(7.0-12.0)$ & & $7.0(7.0-12.0)$ & \\
\hline & & M1c & $4(0.0)$ & $4.0(1.0-18.0)$ & & $4.0(1.0-18.0)$ & \\
\hline & \multirow[t]{3}{*}{$\mathrm{G} 2$} & M1a & $108(1.3)$ & $15.0(9.0-45.0)$ & $<.001$ & $14.0(6.0-32.0)$ & $<.001$ \\
\hline & & M1b & $34(0.4)$ & $13.0(2.0-21.0)$ & & $13.0(2.0-21.0)$ & \\
\hline & & M1c & $63(0.7)$ & $5.0(2.0-12.0)$ & & $4.0(2.0-10.0)$ & \\
\hline & \multirow[t]{3}{*}{ G3 } & M1a & $537(6.3)$ & $15.0(7.0-31.0)$ & $<.001$ & $15.0(7.0-29.0)$ & $<.001$ \\
\hline & & M1b & $174(2.0)$ & $13.0(5.0-20.0)$ & & $12.0(4.0-19.0)$ & \\
\hline & & M1c & 291 (3.4) & $8.0(3.0-14.0)$ & & $7.0(3.0-14.0)$ & \\
\hline
\end{tabular}

Abbreviations: HoR, hormone receptor; G, grade; IQR, interquartile range; NR, not reached. aNo events observed due to limited sample size. 
eTable 9. Multivariate Analysis of Cancer-Specific Mortality by Treatment Modality ${ }^{\mathrm{a}}$

Model 1

Model 2

Model 3

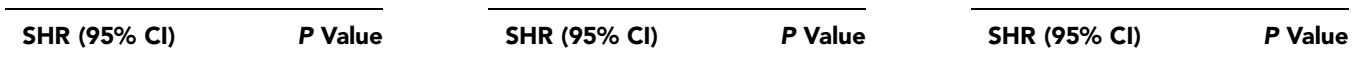

Part I: patients who did not receive RT

M1a

None

Surgery only

Chemo only

Surgery and chemo

M1b

None

Surgery only

Chemo only

Surgery and chemo

M1c

\section{None}

Surgery only

Chemo only

Surgery and chemo
Ref

0.65 (0.53-0.79)

$0.70(0.60-0.83)$

$0.49(0.40-0.59)$

Ref

0.69 (0.49-0.97)

$0.55(0.45-0.69)$

$0.49(0.38-0.63)$

Ref

0.65 (0.47-0.92)

.014

$0.45(0.38-0.54)$

$0.31(0.24-0.39)$

$<.001$

$<.001$

.032

$<.001$

$<.001$

$<.001$

Ref

$1.08(0.88-1.33)$

$0.75(0.60-0.94)$

.462

Ref

.011

$0.69(0.58-0.82)$

$<.001$

Part II: patients with TNBC who did not receive RT

M1a

\section{None}

Ref

Surgery only

$0.94(0.56-1.59)$

Chemo only

$0.62(0.40-0.95)$

$0.47(0.30-0.73)$

Ref

None

$0.78(0.29-2.12)$

$0.27(0.13-0.57)$

.629

.824

.026

$<.001$

$0.27(0.13-0.56)$

$<.001$

$<.001$

Ref

None

$1.50(0.75-3.02)$

.251

Surgery only

$0.48(0.31-0.73)$

$0.21(0.13-0.35)$

$<.001$

$<.001$

Surgery and chemo

629

Ref
$0.65(0.44-0.96)$

0.69 (0.49-0.97)

$0.47(0.32-0.68)$

.035

.207

Ref

$0.81(0.57-1.13)$

.056

$0.88(0.69-1.12)$

.291

Abbreviations: chemo, chemotherapy; SHR, subdistribution hazard ratio; TNBC, triple-negative breast cancer.

aln the multivariate analyses, a different treatment modality was selected as the reference category to perform the multiple comparisons ("no treatment" for Model 1 ,

"surgery only" for Model 2, and "chemo only" for Model 3). Results of univariate analyses are not shown. The competing risk model was adjusted for age, sex, race/ ethnicity, marital status, histologic grade, AJCC T \& N category, molecular subtype, and treatment modality (data not shown). 
eTable 10. Association of Primary Tumor Surgery With Mortality

Univariable Analysis

Multivariable Analysis

\begin{tabular}{|c|c|c|c|c|c|c|c|c|c|}
\hline \multirow{2}{*}{ Local Surgery } & \multirow{2}{*}{$\begin{array}{l}\text { Patients } \\
\text { n (\%) }\end{array}$} & \multirow{2}{*}{\multicolumn{2}{|c|}{ Cancer-Specific Mortality }} & \multirow{2}{*}{\multicolumn{2}{|c|}{ All-Cause Mortality }} & & & & \\
\hline & & & & & & \multicolumn{2}{|c|}{ Cancer-Specific Mortality } & \multicolumn{2}{|c|}{ All-Cause Mortality } \\
\hline No & $5,444(63.4)$ & $1.86(1.72-2.01)$ & $<.001$ & $1.94(1.79-2.10)$ & $<.001$ & $1.59(1.46-1.74)$ & $<.001$ & $1.67(1.53-1.82)$ & $<.001$ \\
\hline Lumpectomy ${ }^{\mathrm{a}}$ & $908(10.6)$ & $0.97(0.85-1.10)$ & .594 & $0.96(0.84-1.09)$ & .514 & $0.98(0.85-1.12)$ & .723 & $0.94(0.82-1.08)$ & .379 \\
\hline Mastectomy ${ }^{b}$ & $2,230(26.0)$ & Ref & & Ref & & Ref & & Ref & \\
\hline
\end{tabular}

The competing risk model was adjusted for age, race/ethnicity, marital status, histologic grade, AJCC T category, molecular subtype, use of chemotherapy, and M1 subcategory. The Cox proportional hazards model was adjusted for age, race/ethnicity, marital status, histologic grade, AJCC T \& N category, molecular subtype, use of chemotherapy, and M1 subcategory.

Abbreviations: HR, hazard ratio; SHR, subdistribution hazard ratio.

alncludes lumpectomy, quadrantectomy, and breast-conserving surgery.

bIncludes subcutaneous mastectomy, simple mastectomy, and modified or extended radical mastectomy. 


\section{eAppendix 1.}

\section{Validation of M1 Category Subdivision System}

The association of M1 subcategories with cancer-specific or all-cause mortality was also observed in the validation and whole sets. The 3-year cumulative incidence rates of cancer-specific mortality increased across M1 subcategories: $44.1 \%$ (95\% CI, 41.6\%-46.7\%) for Mla; 50.6\% (95\% CI, 46.3\%-55.1\%) for M1b; and 70.4\% (95\% CI, $66.4 \%-74.0 \%$ ) for Mlc in the validation set (Figure 1B), and $43.2 \%$ (95\% CI, 41.4\%-45.1\%) for M1a, 52.1\% (95\% CI, 48.9\%-55.1\%) for M1b, and 71.3\% (95\% CI, 68.6\%-73.8\%) for Mlc in the whole set (Figure 1C). After adjusting for clinicopathologic variables, M1 subcategories were associated with higher cancer-specific mortality both in the validation set (M1b vs Mla: SHR, 1.32; 95\% CI, 1.17-1.51; $P<.001$; M1c vs M1a: SHR, 2.15; 95\% CI, 1.92-2.42; $P<.001$ ) and the whole set (M1b vs M1a: SHR, 1.39; 95\% CI, 1.27-1.53; $P<.001$; Mlc vs Mla: SHR, 2.29; 95\% CI, 2.11-2.48; $P<.001$ ) (see Table 2 and supplemental eTable 5). The 3-year all-cause mortality rates for M1 subcategories were $48.7 \%$ (95\% CI, $46.1 \%-51.3 \%$ ) for M1a, $56.8 \%$ (95\% CI, 52.4\%-61.0\%) for Mlb, and 74.7\% (95\% CI, 70.8\%-78.2\%) for Mlc in the validation set and 48.3\% (95\% CI, $46.4 \%-50.1 \%$ ) for Mla, $58.0 \%$ (95\% CI, 54.9\%-61.1\%) for M1b, and 75.9\% (95\% CI, 73.3\%-78.4\%) for M1c in the whole set (Figure 1E-F). After adjusting for age at diagnosis, race/ethnicity, marital status, histologic grade, AJCC T and N stage, molecular subtype, and treatment modality, similar directions of association were observed across M1 subcategories both in the validation set (M1b vs Mla: SHR, 1.35; 95\% CI, 1.20-1.53; $P<.001$; M1c vs Mla: SHR, 2.09; 95\% CI, 1.88-2.33; $P<.001$ ) and the whole set (M1b vs M1a: SHR, 1.41; 95\% CI, 1.29-1.53; $P<.001$; M1c vs M1a: SHR, 2.22; 95\% CI, 2.06-2.41; $P<.001$ ) (see Table 2 and supplemental eTable 6).

Baseline characteristics and treatment information stratified by molecular subtypes are displayed in supplemental eTable 7. M1 subcategories were also associated with different cancer-specific mortality across the molecular subtypes in the whole set. For patients with HoR+/HER2-breast cancer, $51 \%$ and $152 \%$ increased risk were observed for those with Mlb (SHR, 1.51; 95\% CI, 1.34-1.70; $P<.001$ ) and Mlc (SHR, 2.52; 95\% CI, 2.27-2.80; $P<.001$ ) compared with Mla disease. Similar directions of associations were observed across M1 subcategories in patients with HoR $+/$ HER2+ (M1b vs M1a:SHR, 1.37; 95\% CI, 1.07-1.75; $P=.013$; M1c vs Mla: SHR, 2.47; 95\% CI, 2.02-3.01; $P<.001$ ), HoR-/HER2+ (M1b vs M1a: SHR, 1.46; 95\% CI, 1.07-1.98; $P=.017$; M1c vs M1a: SHR, 2.72; 95\% CI, 2.09-3.52; $P<.001$ ), and triple-negative breast cancer (Mlb vs Mla: SHR, 1.39; 95\% CI, 1.15-1.68; $P=.006$; M1c vs M1a: SHR, 2.23; 95\% CI, 1.92-2.60; $P<.001$ ) (see supplemental eFigure 2). Similarly, significantly different median survival was observed across M1 subcategories when stratified by molecular subtypes and histologic grades (see supplemental eTable 8). 